\title{
Post-transcriptional regulation of Leishmania fitness gain
}

Laura Piel ${ }^{1,2}$, K. Shanmugha Rajan ${ }^{3}$, Giovanni Bussotti ${ }^{1,4}$, Hugo Varet ${ }^{4,5}$, Rachel Legendre ${ }^{4,5}$, Caroline Proux ${ }^{5}$, Thibaut Douché ${ }^{6}$, Quentin Giai-Gianetto ${ }^{4,6}$, Thibault Chaze ${ }^{6}$, Barbora Vojtkova ${ }^{7}$, Nadav Gordon-Bar ${ }^{3}$, Tirza Doniger ${ }^{3}$, Smadar Cohen-Chalamish ${ }^{3}$, Praveenkumar Rengaraj ${ }^{3}$, Céline Besse ${ }^{8}$, Anne Boland ${ }^{8}$, Jovana Sadlova ${ }^{7}$, Jean-François Deleuze ${ }^{8}$, Mariette Matondo ${ }^{6}$, Ron Unger ${ }^{3}$, Petr Volf ${ }^{7}$, Shulamit Michaeli ${ }^{3}$, Pascale Pescher ${ }^{1, *}$ and Gerald F. Späth ${ }^{1, *}$

${ }^{1}$ Institut Pasteur, INSERM U1201, Unité de Parasitologie moléculaire et Signalisation, 75015 Paris, France; ${ }^{2}$ Université de Paris, 75013 Paris, France; ${ }^{3}$ The Mina and Everard Goodman Faculty of Life Sciences and Advanced Materials and Nanotechnology Institute, Bar-llan University, Ramat-Gan 52900, Israel; ${ }^{4}$ Institut Pasteur, Bioinformatics and Biostatistics Hub, Department of Computational Biology, USR 3756 IP CNRS, 75015 Paris, France; Institut Pasteur, Biomics, 75015 Paris, France; Institut Pasteur, UTechS MSBio, 75015 Paris, France; ${ }^{6}$ Institut Pasteur, Proteomics Platform Mass Spectrometry for Biology UTechS, C2RT, USR2000 CNRS, 75015 Paris, France; ${ }^{7}$ Department of Parasitology, Faculty of Science, Charles University, Prague 2, 128 44, Czech Republic; ${ }^{8}$ Université Paris-Saclay, CEA, Centre National de Recherche en Génomique Humaine, 91057, Evry, France

*co-corresponding authors: gerald.spaeth@pasteur.fr, pascale.pescher@pasteur.fr

Keywords: Leishmania, experimental evolution, fitness, genomics, transcriptomics, posttranscriptional adaptation, small nucleolar RNAs 


\section{Abstract}

The protozoan parasite Leishmania donovani causes fatal human visceral leishmaniasis in absence of treatment. Genome instability has been recognized as a driver in Leishmania fitness gain in response to environmental change or chemotherapy. How genome instability generates beneficial phenotypes despite potential deleterious gene dosage effects is unknown. Here we address this important open question applying experimental evolution and integrative systems approaches on parasites adapting to in vitro culture. Phenotypic analyses of parasites from early and late stages of culture adaptation revealed an important fitness tradeoff, with selection for accelerated growth (fitness gain) impairing infectivity (fitness costs). Comparative genomics, transcriptomics and proteomics analyses revealed a complex regulatory network driving parasite fitness, with genome instability causing highly reproducible, gene dosage-dependent changes in protein linked to post-transcriptional regulation. These in turn were associated with a gene dosage-independent reduction in flagellar transcripts and a coordinated increase in abundance of coding and non-coding RNAs known to regulate ribosomal biogenesis and protein translation. We correlated differential expression of small nucleolar RNAs (snoRNAs) with changes in rRNA modification, providing first evidence that Leishmania fitness gain may be controlled by post-transcriptional and epitranscriptomic regulation. Our findings propose a novel model for Leishmania fitness gain, where differential regulation of mRNA stability and the generation of fitness-adapted ribosomes may potentially filter deleterious from beneficial gene dosage effects and provide proteomic robustness to genetically heterogenous, adapting parasite populations. This model challenges the current, genome-centric approach to Leishmania epidemiology and identifies the Leishmania non-coding small RNome as a potential novel source for biomarker discovery. 
bioRxiv preprint doi: https://doi org/10.1101/2021.03.22.436378; this version posted March 22, 2021. The copyright holder for this preprint (which was not certified by peer review) is the author/funder, who has granted bioRxiv a license to display the preprint in perpetuity. It is made available under aCC-BY-NC-ND 4.0 International license.

\section{Introduction}

Parasitic protozoa of the genus Leishmania are the etiologic agents of a spectrum of severe diseases known as leishmaniases that cause substantial human morbidity and are among the five most serious parasitic diseases worldwide. Today, almost 1 billion people are at risk of Leishmania infection in close to 100 endemic countries throughout tropical and subtropical regions, with over 12 million people diagnosed with the infection [1]. Leishmaniasis represents a global public health challenge: recurrent epidemics are observed in South America, the Maghreb, Middle East, East Africa and India, and Leishmania infection has been declared an emerging disease in the EU and South East Asia [1, 2]. In absence of treatment, visceral leishmaniasis (VL - also known as Kala Azar) is the most severe and fatal form of the disease, caused either by Leishmania (L.) donovani or L. infantum.

Most Leishmania species show a digenic life cycle comprising two major developmental stages that infect two distinct hosts. The motile, extracellular promastigote form of Leishmania proliferates inside the digestive tract of the sand fly insect vector, while the non-motile, intracellular amastigote form develops and proliferates inside fully acidified, macrophage phagolysosomes of mammalian hosts. Aside stage differentiation, the success of Leishmania as a pathogenic microbe relies on its capacity to adapt to a variety of environmental fluctuations encountered in their hosts via an evolutionary process. Evolutionary adaptation relies on the classical Darwinian paradigm, where spontaneous mutations and stochastic changes in gene expression generate genetically and phenotypically heterogenous populations that compete for reproductive success in a given environment, thus driving natural selection of the fittest individuals [3]. While this process is well understood in viral and bacterial infections, only little information is available on evolutionary adaptation of eukaryotic pathogens, notably protozoan parasites. This is especially relevant to trypanosomatids, which - in contrast to classical eukaryotes - do not regulate expression of protein coding genes by transcriptional control. Transcription of protein coding genes in these earlybranching eukaryotes is constitutive, with genes being arranged in long, polycistronic transcription units, and mature mRNAs being generated from precursors via a highly parasite-specific, transsplicing process that is linked to polyadenylation $[4,5]$. In the absence of classical transcriptional regulation [5], Leishmania has evolved and emphasized other forms of control, notably regulation of RNA abundance by post-transcriptional regulation and gene dosage variation [6-10].

A hallmark of Leishmania biology is the intrinsic plasticity of its genome, with frequent copy number variations (CNVs) of individual genes or chromosomes linked to drug resistance or changes in tissue tropism [7, 11-16]. Combining experimental evolution and comparative genomics approaches, we recently linked both forms of genome instability with fitness gain in vitro. DNA read depth 
analysis of the genomes of $L$. donovani parasites evolving to higher fitness in culture identified amplification of a series of chromosomes as highly reproducible drivers of early culture adaptation [10]. Long-term adaptation in contrast was linked to the positive selection of gene copy number variants, which were amplified as part of functionally related, epistatic networks that allowed the emergence of phenotypes linked to ribosomal biogenesis, translation and proliferation (Bussotti et al., in preparation). Leishmania genomic adaptation thus occurs through a two-stage process reminiscent to other fast-growing eukaryotic cells (e.g. fungi and cancer cells $[17,18]$ ), involving short-term adaptation by karyotypic changes and long-term adaptation through slower gene CNVs $[10,19]$ (Bussotti et al., in preparation).

Together these reports draw a complex picture of Leishmania fitness gain and raise a series of important new questions on (i) the nature of the genes that drive positive selection of the observed karyotypic changes during fitness gain in vitro, (ii) the potential fitness costs associated with karyotypic adaptation, and (iii) the mechanisms evolved by the parasite to harness genome instability for fitness gain and compensate for deleterious gene dosage effects. Here we combined experimental evolution and integrative systems approaches to address these questions and gain novel insight into regulatory mechanisms underlying Leishmania fitness gain during adaptation to culture. Our analyses reveal mechanisms at gene, transcript and protein levels that harness genome instability for fitness gain through gene dosage-dependent changes that affect post-transcriptional regulation and gene dosage-independent changes in epitranscriptomic control and ribosomal biogenesis. 


\section{Material and Methods}

\section{$\underline{\text { Animals and ethics statement }}$}

Six to eight-week-old, female mice (Mus musculus, C57BL/6JRj) and 5 female Golden Syrian hamsters (Mesocricetus auratus RjHan:AURA, weighting between $60-70 \mathrm{~g}$ ) were purchased from Janvier Laboratories. All animals were handled under specific, pathogen-free conditions in biohazard level 3 animal facilities (A3) accredited by the French Ministry of Agriculture for performing experiments on live rodents (agreement A75-15-01). Work on animals was performed in compliance with French and European regulations on care and protection of laboratory animals (EC Directive 2010/63, French Law 2013-118, February 6th, 2013). All animal experiments were approved by the Ethics Committee and the Animal welfare body of Institut Pasteur (dha190013 and 180091) and by the Ministère de I’Enseignement Supérieur, de la Recherche et de l'Innovation (project $n^{\circ} \# 19683$ ).

\section{Parasites and culture}

Leishmania donovani strain 1S2D (MHOM/SD/62/1S-CL2D) was obtained from Henry Murray, Weill Cornell Medical College, New York, USA and maintained by serial passages in hamsters. Amastigotes were recovered from infected hamster spleen and differentiated into promastigotes in M199 complete medium (M199, 10\% FBS, 20 mM HEPES; $100 \mu \mathrm{M}$ adenine, $2 \mathrm{mM}$ L-glutamine, $10 \mu \mathrm{g} / \mathrm{ml}$ folic acid, $13.7 \mu \mathrm{M}$ hemin, $4.2 \mathrm{mM} \mathrm{NaHCO}$, 1xRPMI1640 vitamins, $8 \mu \mathrm{M}$ 6-biopterin, 100 units penicillin and $100 \mathrm{\mu g} / \mathrm{ml}$ streptomycin, $\mathrm{pH}$ 7.4) at $26^{\circ} \mathrm{C}$. Promastigotes, derived from splenic amastigotes, were serially passaged once stationary phase was reached for less than 5 passages (EP, early passage) or 20 passages (LP, late passage) corresponding to $\sim 20$ and 190 generations, respectively. Strains issued from independent experimental evolution assays are identified by number (i.e. EP.1 and LP.1 are the strains resulting from experiment 1).

\section{Parasite growth and determination of the generation time}

Promastigotes in exponential growth phase were seeded at $2 \times 10^{6}$ (EP) or $1 \times 10^{5}$ (LP) parasites per $\mathrm{ml}$ in M199 complete medium. Parasites were counted every 24 hours during a three-day period at logarithmic growth phase, and the generation time was calculated according to the formula doubling time $=\frac{t \times \log (2)}{\log C(t)-\log C(i)}$. Experiments were performed in triplicates and statistical significance was assessed by t-test.

\section{$\underline{\text { Ficoll gradient centrifugation for metacyclic promastigote enrichment }}$}

Promastigote cultures at day 3 of stationary culture phase were collected and adjusted to $3 \times 10^{8}$ cells/ml. Ficoll PM400 (GE Healthcare) was used to prepare a $20 \%$ stock solution in PBS and diluted 
bioRxiv preprint doi: https://doi org/10.1101/2021.03.22.436378; this version posted March 22, 2021. The copyright holder for this preprint (which was not certified by peer review) is the author/funder, who has granted bioRxiv a license to display the preprint in perpetuity. It is made available under aCC-BY-NC-ND 4.0 International license.

for preparation of $10 \%$ and $5 \%$ Ficoll solutions. Four $\mathrm{ml}$ of $10 \%$ Ficoll were overlaid by $4 \mathrm{ml}$ of $5 \%$ Ficoll and $4 \mathrm{ml}$ of parasite suspension were layered on top of the Ficoll cushion. Tubes were centrifuged at 1,300 $\times \mathrm{g}$ for $15 \mathrm{~min}$ at room temperature without brake. The metacyclic-enriched fractions were recovered at the interface between the $10 \%$ and $5 \%$ Ficoll layers. Parasites were washed with PBS and adjusted to the final concentration required for a given experiment.

\section{DNA and RNA extraction and sequencing}

The different growth kinetics between EP and LP parasites were considered when recovering parasites from logarithmic growth phase (i.e. using the same density), and stationary growth phase (i.e. using parasites kept for the same time at this phase) for DNA or RNA preparation. DNA was prepared from parasites in exponential culture phase. EP.1 and LP.1, LP.6 and LP.7 promastigotes were centrifuged at $1,600 \times g$ for $10 \mathrm{~min}$ at room temperature. Approximately $1 \times 10^{8}$ promastigotes from logarithmic growth phase were re-suspended in $200 \mu \mathrm{l}$ PBS and genomic DNA was purified using DNeasy Blood and Tissue kit from Qiagen and RNase A according to the manufacturer's instructions. DNA concentrations were measured in duplicate by fluorescence using a Molecular Device fluorescence plate reader (Quant-IT kits, Thermo Fischer Scientific). The quality of the DNAs was controlled determining the DNA Integrity Number (DIN) analyzing 20 ng of DNA on a TapeStation 4200 (Agilent). One $\mu \mathrm{g}$ genomic DNA was used to prepare a library for whole genome sequencing on an automated platform, using the Illumina "TruSeq DNA PCR-Free Library Preparation Kit", according to the manufacturer's instructions. After normalization and quality control, qualified libraries were sequenced on a HiSeqX5 platform from Illumina (Illumina Inc., CA, USA) at the Centre National de Recherche en Génétique Humaine (CEA, Evry, France), generating paired-ended, 150-bp reads. Sequence quality parameters were assessed throughout the sequencing run. Standard bioinformatics analysis of sequencing data was based on the Illumina pipeline to generate a FASTQ file for each sample.

Total RNA was extracted from EP.1 and LP.1 cultures at logarithmic and stationary ( 3 days) culture phases or from EP.1 metacyclic-enriched parasites (EP.1 meta). Promastigotes were centrifuged at $3,000 \times \mathrm{g}$ for $10 \mathrm{~min}$ at room temperature and re-suspended in the lysis buffer supplied with the Qiagen RNeasy Plus kit. The samples were stored at $-80^{\circ} \mathrm{C}$ and RNA extractions were performed according to the manufacturer's instructions, including a DNase treatment. RNA integrity was controlled using the Agilent Bioanalyzer. DNAse-treated RNA extracts were used for library preparation using the Truseq Stranded mRNA sample preparation kit (Illumina, San Diego, California) according to the manufacturer's instructions. An initial poly (A+) RNA isolation step (included in the Illumina protocol) was performed on total RNA to remove ribosomal RNA. Fragmentation was 
performed on the enriched fraction by divalent ions at high temperature. The fragmented RNA samples were randomly primed for reverse transcription followed by second-strand synthesis to create double-stranded cDNA fragments. No end repair step was necessary. An adenine was added to the 3 '-end and specific Illumina adapters were ligated. Ligation products were submitted to PCR amplification. The obtained oriented libraries were controlled by Bioanalyzer DNA1000 Chips (Agilent, \# 5067-1504) and quantified by spectrofluorimetry (Quant-iT ${ }^{\mathrm{TM}}$ High-Sensitivity DNA Assay Kit, \#Q33120, Invitrogen). Sequencing was performed on the Illumina Hiseq2500 platform at the Biomics Center (Institut Pasteur, Paris, France) to generate single-ended, 130-bp reads bearing strand specificity.

For transcriptome-wide mapping of pseudouridine sites ( $\Psi$-seq), total RNA was either untreated or treated with $\mathrm{N}$-cyclohexyl-N- $\beta$-(4-methylmorpholinium) (CMC) in bicine buffer (0.17 M CMC in 50 $\mathrm{mM}$ bicine, $\mathrm{pH}$ 8.3, $4 \mathrm{mM}$ EDTA, $7 \mathrm{M}$ urea) at $37^{\circ} \mathrm{C}$ for $20 \mathrm{~min}$. Excess $\mathrm{CMC}$ was removed by ethanol precipitation. To remove $\mathrm{CMC}$ groups attached to $\mathrm{G}$ and $\mathrm{U}$, the $\mathrm{CMC}$-treated RNA was subjected to alkaline hydrolysis with $\mathrm{Na}_{2} \mathrm{CO}_{3}(50 \mathrm{mM}, \mathrm{pH} 10.4)$ at $37^{\circ} \mathrm{C}$ for $4 \mathrm{~h}$, as previously described [20-23]. The reacted RNA was recovered by phenol chloroform extraction, and ethanol precipitation. An adaptor was ligated to the $3^{\prime}$ end of the total RNA (upon fragmentation) before and after CMC treatment, and cDNA was prepared using AffinityScript reverse transcriptase (Agilent). The cDNA was then ligated to an adaptor, PCR amplified, and the samples were sequenced in an Illumina NextSeq machine in paired-end mode, 42-bp reads (20 million reads for each sample).

For the preparation of the small RNome, whole cell extracts were prepared from L. donovani EP and

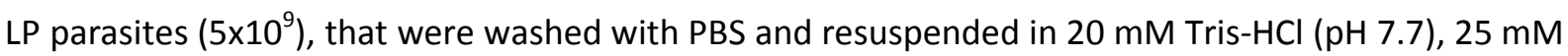
$\mathrm{KCl}$, and $10 \mathrm{mM} \mathrm{MgCl}_{2}$, were equilibrated in a nitrogen cavitation bomb (Parr Instruments Co.) with $750 \mathrm{psi} \mathrm{N} 2$ for $1 \mathrm{~h}$ at $4^{\circ} \mathrm{C}$, and disrupted by release from the bomb. After nitrogen cavitation, the ribonucleoproteins (RNPs) were extracted with $0.4 \mathrm{M} \mathrm{KCl}$. Ribosomes were removed by centrifugation for $3 \mathrm{~h}$ at $35,000 \mathrm{rpm}$ in a Beckman $70.1 \mathrm{Ti}$ rotor $(150,000 \mathrm{xg})$ and the supernatant was defined as post-ribosomal supernatant (PRS). RNA was extracted after treatment with $100 \mu \mathrm{g} / \mathrm{ml}$ of Proteinase $\mathrm{K}, 1 \%$ SDS in the presence of $100 \mu \mathrm{g} / \mathrm{ml}$ DNasel and was used for library preparation as described previously [21]. The samples were sequenced in an Illumina NextSeq machine in pairedend mode, 42-bp reads (40 million reads for each sample).

\section{Protein extraction, digestion and LC-MS/MS acquisition}

Exponentially growing EP.2, EP.3, EP.4, EP.5 and LP.2, LP.3, LP.4 and LP.5 promastigotes were centrifuged at $1600 \times g$ for $10 \mathrm{~min}$ at $4^{\circ} \mathrm{C}$ and washed three times with cold PBS. Parasite lysates were prepared in $8 \mathrm{M}$ urea, $50 \mathrm{mM}$ Tris, supplemented with a protease inhibitor cocktail (cOmplete ${ }^{\mathrm{TM}}$ from 
Roche) and a phosphatase inhibitor cocktail (PhosStop from Roche), $1 \mathrm{ml}$ of lysis buffer per $1.5 \times 10^{9}$ promastigotes. After $10 \mathrm{~min}$ incubation at $4^{\circ} \mathrm{C}$ followed by sonication for $5 \mathrm{~min}$ (sequence of $10 \mathrm{~s}$ pulse and 20s pause) the lysates were centrifuged $15 \mathrm{~min}$ at $14,000 \times \mathrm{g}, 4^{\circ} \mathrm{C}$ and the supernatant was collected and stored at $-80^{\circ} \mathrm{C}$ until use. Proteins were quantified by RC DC ${ }^{\mathrm{TM}}$ protein assay (Bio-Rad) and a control of the protein pattern of all the extracts was performed by SDS-PAGE and silver staining. All the biological samples were further processed for MS-based proteomics approach, data acquisition, and statistical analyses.

Biological samples were adjusted to $1.3 \mu \mathrm{g} \cdot \mu \mathrm{I}^{-1}$ in lysis buffer. Disulfide bridges were reduced in $5 \mathrm{mM}$ DTT (Sigma - 43815) for $30 \mathrm{~min}$ and alkylated in $20 \mathrm{mM}$ iodoacetamide (Sigma - I1149) for $30 \mathrm{~min}$ at room temperature in the dark. Protein samples were diluted 10 fold in $50 \mathrm{mM} \mathrm{Tris-HCl}$ and digested with Sequencing Grade Modified Trypsin (Promega - V5111) at a Protein:Trypsin ratio 50:1 overnight. Then a second digestion was performed to complete this step. Proteolysis was stopped by adding formic acid (FA, Fluka - 94318) at a 1\% final concentration. Resulting peptides were desalted using Sep-Pak SPE cartridge (Waters) according to the manufacturer's instructions. Peptides were concentrated to almost dryness and were resuspended in 2\% Acetonitrile (ACN) / $0.1 \%$ FA just before LC-MS/MS injection.

All analyses were performed on a Q Exactive ${ }^{\mathrm{TM}}$ Plus Mass Spectrometer (Thermo Fisher Scientific) coupled with a Proxeon EASY-nLC 1000 (Thermo Fisher Scientific). One $\mu \mathrm{g}$ of peptides was injected into a home-made $50 \mathrm{~cm} \mathrm{C18}$ column (1.9 $\mu \mathrm{m}$ particles, $100 \AA ̊$ pore size, ReproSil-Pur Basic C18, Dr. Maisch $\mathrm{GmbH}$, Ammerbuch-Entringen, Germany). Column equilibration and peptide loading were performed at 900 bars in buffer A (0.1\% FA). Peptides were separated with a multi-step gradient of 2 to $5 \%$ buffer B ( $80 \%$ ACN, $0.1 \%$ FA) for $5 \mathrm{~min}, 5$ to $22 \%$ buffer B for $150 \mathrm{~min}, 22$ to $45 \%$ buffer B for 60 min, 45 to $80 \%$ buffer B for $10 \mathrm{~min}$ at a flow rate of $250 \mathrm{~nL} / \mathrm{min}$ over $240 \mathrm{~min}$. Column temperature was set to $60^{\circ} \mathrm{C}$. MS data were acquired using Xcalibur software using a data-dependent method. MS scans were acquired at a resolution of 70,000 and MS/MS scans (fixed first mass $100 \mathrm{~m} / \mathrm{z}$ ) at a resolution of 17,500 . The AGC target and maximum injection time for the survey scans and the $\mathrm{MS} / \mathrm{MS}$ scans were set to $3 \mathrm{E}^{6}, 20 \mathrm{~ms}$ and $1 \mathrm{E}^{6}, 60 \mathrm{~ms}$ respectively. An automatic selection of the 10 most intense precursor ions was activated (Top 10) with a 45s dynamic exclusion. The isolation window was set to $1.6 \mathrm{~m} / \mathrm{z}$ and normalized collision energy fixed to 28 for HCD fragmentation. We used an underfill ratio of $1.0 \%$ corresponding to an intensity threshold of $1.7 \mathrm{E}^{5}$. Unassigned precursor ion charge states as well as $1,7,8$ and $>8$ charged states were rejected and peptide match was disabled. 
bioRxiv preprint doi: https://doi org/10.1101/2021.03.22.436378; this version posted March 22, 2021. The copyright holder for this preprint (which was not certified by peer review) is the author/funder, who has granted bioRxiv a license to display the preprint in perpetuity. It is made available under aCC-BY-NC-ND 4.0 International license.

WGS analysis: Genomic DNA reads were aligned to the L. donovani Ld1S reference genome (Bussotti \& Myler unpublished, https://www.ncbi.nlm.nih.gov/bioproject/PRJNA396645) with BWA mem (version 0.7.12) with the flag $-\mathrm{M}$ to mark shorter split hits as secondary. Samtools fixmate, sort, and index (version 1.3) were used to process the alignment files and turn them into bam format [24]. RealignerTargetCreator and IndelRealigner from the GATK suite were run to homogenize indels [25]. Eventually, PCR and optical duplicates were labeled with Picard MarkDuplicates [version 1.94(1484)] (https://broadinstitute.github.io/picard/) using the option "VALIDATION_STRINGENCY=LENIENT". For each read alignment file, Samtools view (version 1.3) and BEDTools genomecov (version 2.25.0) were used to measure the sequencing depth of each nucleotide [26]. Samtools was run with options "-q 50 -F 1028" to discard reads with a low map quality score or potential duplicates, while BEDTools genomecov was run with options "-d -split." The chromosome sequencing coverage was used to evaluate aneuploidy between EP.1 and LP.1 samples. For this purpose the nucleotide coverage was normalized by the median genomic coverage. Then for each sample and for each chromosome, the median sequencing coverage was computed for contiguous windows of 2,500 bases. Eventually the window coverage was further normalized by chromosome 36 median coverage and multiplied by two. The gene counts were produced using featureCounts (version 1.4.6-p3 [27]) with these parameters: -s 0 -t gene -g gene_id and were normalized according to the median-ratio method.

RNAseq analysis: For total RNAseq data, the bioinformatics analysis was performed using the RNAseq pipeline from Sequana [28]. Reads were cleaned of adapter sequences and low-quality sequences using cutadapt version 1.11 [29]. Only sequences of at least 25 nucleotides in length were considered for further analysis. STAR version 2.5.0a [30], with default parameters, was used for alignment on the reference genome (GCA 002243465.1). Genes were counted using featureCounts version 1.4.6-p3 [27] from Subreads package (parameters: -t gene -g gene_id -s 1). Count data were analyzed using R version 3.6.1 [31] and the Bioconductor package DESeq2 version 1.24.0 [32]. The normalization and dispersion estimation were performed with DESeq2 using the default parameters and statistical tests for differential expression were performed applying the independent filtering algorithm. A generalized linear model including the replicate effect as blocking factor was set in order to test for the differential expression between EP.1 log, EP.1 stat, EP.1 meta, LP.1 log and LP.1 stat. For each pairwise comparison, raw p-values were adjusted for multiple testing according to the Benjamini and Hochberg (BH) procedure [33] and genes with an adjusted p-value lower than 0.05 were considered differentially expressed. The RNAseq data have been deposited in NCBI's Gene Expression Omnibus (Edgar et al., 2002) and are accessible through GEO Series accession number GSE165615 (https://www.ncbi.nlm.nih.gov/geo/query/acc.cgi?acc=GSE165615). 
bioRxiv preprint doi: https://doi org/10.1101/2021.03.22.436378; this version posted March 22, 2021. The copyright holder for this preprint (which was not certified by peer review) is the author/funder, who has granted bioRxiv a license to display the preprint in perpetuity. It is made available under aCC-BY-NC-ND 4.0 International license.

For small RNome analysis, $\Psi$-seq and detection of pseudouridylated sites, the 42 bp sequence reads obtained from the Illumina Genome Analyzer were first trimmed of Illumina adapters using the FASTX toolkit (http://hannonlab.cshl.edu/fastx toolkit), and reads of 15 nucleotides or less were discarded from subsequent analysis. The remaining reads were mapped to the reference genome (GCA 002243465.1) using SMALT v0.7.5 (https://www.sanger.ac.uk/tool/smalt-0/) with the default parameters. Only properly paired partners were retained. Each read pair was "virtually" extended to cover the area from the beginning of the first read to the end of its partner. For each base, the number of reads initializing at that location as well as the number of reads covering the position $\begin{array}{llllll}\text { were } & \text { calculated. } & \text { combination } & \text { of } & \text { BEDTools } & \text { v2.26.0 }\end{array}$ (http://bedtools.readthedocs.io/en/latest/) and in-house Perl scripts was used to calculate the $\Psi$ ratio and $\Psi$-fc (fold change), as previously described [21, 22].

Proteomics analysis: Raw data were analyzed using MaxQuant software version 1.5.3.8 [34] using the Andromeda search engine [35]. The MS/MS spectra were searched against the Ld1S database (Bussotti et al., in preparation). The settings for the search included (i) trypsin digestion with a maximum of two missed cleavages, (ii) variable modifications for methionine oxidation and $\mathrm{N}$ terminal acetylation, and (iii) fixed modification for cysteine carbamidomethylation. The minimum peptide length was set to 7 amino acids and the false discovery rate (FDR) for peptide and protein identification was set to 0.01 . The main search peptide tolerance was set to $4.5 \mathrm{ppm}$ and to $20 \mathrm{ppm}$ for the MS/MS match tolerance. The setting 'second peptides' was enabled to identify cofragmentation events. Quantification was performed using the XIC-based Label-free quantification (LFQ) algorithm with the Fast LFQ mode as previously described [36]. Unique and razor peptides, including modified peptides, with at least two ratio counts were accepted for quantification. The mass spectrometry proteomics data were deposited to the ProteomeXchange Consortium via the PRIDE partner repository with the dataset identifier PXD020236 [37].

For the differential analyses, proteins categorized as 'reverse', 'contaminant' and 'only identified by site' were discarded from the list of identified proteins. After log2 transformation, LFQ values were normalized by median centering within conditions (normalizeD function of the $\mathrm{R}$ package DAPAR [38]). Remaining proteins without any LFQ value in one of the conditions (either EP or LP) and at least two values in the other condition were considered as exclusively expressed proteins. Missing values across the four biological replicates were imputed using the imp.norm function of the R package norm (norm: Analysis of multivariate normal datasets with missing values. $2013 \mathrm{R}$ package version 1.0-9.5). A limma t test was applied to determine proteins with a significant difference in abundance while imposing a minimal fold change of 2 between the conditions to conclude that they are differentially abundant $[39,40]$. An adaptive Benjamini-Hochberg procedure was applied on the 
resulting $p$-values using the function adjust.p of $\mathrm{R}$ package $c p 4 p[41]$ and the robust method described in Pounds et al. [42] to estimate the proportion of true null hypotheses among the set of statistical tests. The proteins associated to an adjusted p-value inferior to a False Discovery Rate (FDR) of 0.01 have been considered as significant and differentially abundant proteins.

Gene Ontology (GO)-enrichment analyses: The Biological Networks Gene Ontology tool (BiNGO) plugin of the Cytoscape software package (version 3.8.2) was used. A Benjamini \& Hochberg false discovery rate with a significance level of 0.05 was applied. The lists of $L$. donovani GO terms were built in house. In order to assign the Gene Ontology Identifiers (GO IDs) we combined the GO-derived identifiers with the ones available from the corresponding orthologs in target species: LdBPK, $L$. infantum, L. major, L. mexicana, Typanosoma brucei brucei 927 (Tbru) and Typanosoma cruzi (Tcru). For each target species we retrieved both the "curated" and "computed" GO IDs from TriTrypDB on the 11/09/2019. OrthoFinder with the DIAMOND search program was applied to establish orthology between the genes in Ld1S and in target species. In "one-to-many" orthology relations we concatenated all the non-redundant GO IDs from all the homologs. The GO IDs were then assigned based on the hierarchy: LdBPK curated $>$ LdBPK GO $>L$. infantum curated $>L$. major curated $>L$. mexicana curated $>$ Tbru curated $>$ Tcru curated $>$ LdBPK computed $>$ L. infantum computed $>L$. major computed $>$ L. mexicana computed $>$ Tbru computed $>$ Tcru computed. The GO IDs were assigned if not present in any higher rank GO ID data set. The GO IDs of snoRNAs, UsnRNA, SLRNA and 7SL classes defined by homology with L. major Friedlin genes were manually attributed. Overall, we assigned biological process (BP), molecular function (MF) and cellular component (CC) GO IDs to $5,246,4,521$ and 7,236 Ld1S genes.

\section{$\underline{\text { Northern blot analyses }}$}

Total RNA extracted from EP and LP cells $(10 \mu \mathrm{g})$ were separated on $10 \%$ acrylamide denaturing gels, transferred to nitrocellulose membranes and analyzed by autoradiography. RNA probes were prepared by in vitro transcription using $\alpha{ }^{32}$ P-UTP [21]. Three independent northern blots were performed.

\section{Bone Marrow Derived Macrophages and infection}

Bone marrow exudate cells were recovered from tibias and femurs of C57BL/6JRj female mice (Janvier Labs) and macrophages differentiated in DMEM complete medium (DMEM, 15\% FBS, 10 mM HEPES, $50 \mu \mathrm{M}$ 2-mercaptoethanol, 50 units of penicillin and $50 \mu \mathrm{g} / \mathrm{ml}$ of streptomycin) supplemented with $75 \mathrm{ng} / \mathrm{ml}$ of recombinant mouse colony stimulating factor-1 (rmCSF-1, ImmunoTools) [43]. A total of $1.5 \times 10^{5}$ Bone Marrow Derived Macrophages (BMDMs) were plated on glass coverslips in 24wells plates and incubated overnight at $37^{\circ} \mathrm{C}, 5 \% \mathrm{CO}_{2}$ prior to Leishmania infection. 
Promastigotes from stationary culture phase or metacyclic-enriched parasite fractions were pelleted by centrifugation at $3,000 \times g$ for $10 \mathrm{~min}$ at room temperature and re-suspended in PBS. The concentration was adjusted to $6 \times 10^{7}$ parasites per $\mathrm{ml}$ and $50 \mu \mathrm{l}$ were added to the BMDM cultures at a multiplicity of infection (MOI) of 20 parasites per 1 macrophage. Plates were centrifuged at $300 \times g$ for $5 \mathrm{~min}$ at room temperature to allow for a faster sedimentation of the parasites onto the macrophage monolayer. After $2 \mathrm{~h}$ of contact, coverslips were washed by successive baths in prewarmed PBS to remove extracellular parasites and transferred into new 24-wells plates containing fresh pre-warmed DMEM culture medium supplemented with $30 \mathrm{ng} / \mu \mathrm{l}$ of rmCSF-1. At 4, 24, 48 and $168 \mathrm{~h}$ post-infection, cells were fixed in $4 \%$ paraformaldehyde (Electron Microscopy Science) and macrophage and parasite nuclei were stained with Hoechst 33342. Images were acquired using a Zeiss Apotome microscope at 40x magnification connected to an Axiocam camera. All the infections were performed in triplicates and at least 100 macrophages were counted per coverslip. The total numbers of infected and non-infected macrophages were recorded and the percentage of infection, the number of parasites per 100 cells and the number of parasites per infected macrophages was calculated and normalized to the values obtained at the initial 4-hour time point. The experiment was performed three times.

\section{Morphological analyses}

Parasites were seeded on poly L-lysine treated coverslips and fixed in $2.5 \%$ glutaraldehyde. Coverslips were mounted on glass slides using Mowiol $^{\circledR}$ 4-88 (Sigma-Aldrich). Images were acquired using an Axiophot microscope at $63 x$ magnification and an Andor camera. Length and width of the parasite cell body, and flagellum length were measured for at least 200 promastigotes using the Image J Fidji software package (https://imagej.net/). The ratios flagellum over body length and body length over body width were determined for the 200 parasites and the Kruskal-Wallis test was used for statistical analysis. The experiment was performed in duplicate.

\section{Sand fly infection}

The colony of Phlebotomus orientalis (originating from Ethiopia), the natural vector of L. donovani, was maintained in the insectary of the Department of Parasitology, Charles University in Prague, under standard conditions $\left(26^{\circ} \mathrm{C}\right.$ on $50 \%$ sucrose and $14 \mathrm{~h}$ light/10h dark photoperiod) as described previously $[44,45]$.

Promastigotes from logarithmic-phase cultures (day 3-4 in culture) were washed twice in saline solution and resuspended in heat-inactivated rabbit blood at a concentration of $1 \times 10^{6}$ promastigotes $/ \mathrm{ml}$. Sand fly females (5-9 days old) were infected by feeding through a chick-skin membrane (BIOPHARM) on a promastigote-containing suspension. Engorged sand flies were 
separated and maintained under the same conditions as the colony. On day 8 post blood meal (PBM), 150 sand fly females were dissected. The thoracic parts and abdominal parts of infected guts were collected separately and pooled together into two samples: thoracic parts of gut (TP) and abdominal parts of gut (AP). The exact numbers of all parasite stages were calculated using a Burker apparatus and the proportion of metacyclic forms was identified on a Giemsa-stained smears separately for TP and AP. Leishmania with flagellar length $<2$ times body length were scored as procyclic forms and those with flagellar length $\geq 2$ times body length as metacyclic forms [46]. 


\section{Results}

\section{L. donovani culture adaptation causes a fitness tradeoff between proliferation and infectivity. In} microbial culture, fitness gain (defined as reproductive capacity) largely equals the level of cell proliferation. Adaptation to in vitro growth thus represents a simple experimental system to assess mechanisms underlying fitness gain. Here we applied such an experimental evolution approach on $L$. donovani amastigotes isolated from infected hamster spleen. Derived promastigotes at early-passage (EP.1) and late-passage (LP.1) were monitored for growth and infectivity with the aim to assess regulatory mechanisms underlying fitness gain and fitness cost observed during culture adaptation. Analyzing cell growth during promastigote culture adaptation revealed robust fitness gain as judged by the reduction in generation time from $13.76+/-1.18$ hours for EP.1 to $9.76+/-0.93$ hours for LP.1 promastigotes (Figure 1A). We next evaluated fitness of these parasites in intracellular macrophage infection, where reproductive success depends on parasite resistance to host cell cytolytic activities, amastigote differentiation and proliferation. BMDMs were incubated with EP.1 and LP.1 promastigotes from day-3 stationary culture (referred to as EP.1 stat and LP.1 stat) and intracellular growth was monitored microscopically for 7 days as previously described [47]. Even though the number of EP.1 stat and LP.1 stat intracellular parasites decreased over the first 24h post-infection, only EP.1 parasites recovered and established persistent infection, while the number of LP.1 parasites steadily declined during the subsequent 6 days (Figure 1B and Figure S1A). Together these results document a fitness tradeoff occurring during $L$. donovani culture adaptation, with accelerated in vitro growth causing virulence attenuation.

We then tested if the fitness cost of LP.1 stat in infectivity was due to a differentiation defect of infectious metacyclic promastigotes. Ficoll gradient centrifugation [48] indeed revealed a 5.5-fold reduction in the number of metacyclic parasites from $3.8 \%$ in EP.1 stat to $0.69 \%$ in LP.1 stat cultures (Figure 1C), which were in addition compromised to establish macrophage infection (Figure 1D and S1B). These results document that the fitness cost in LP.1 meta not only affects the quantity but also the quality of differentiating metacyclic parasites. This was further confirmed by their atypical morphology that was significantly different to bona fide, sand fly-isolated metacyclic promastigotes (Figure S2A), corresponding to leptomonad-like forms as judged by flagellum/body-length ratio and body shape $[49,50]$ (Figure 1E, Figure S2A-C). Surprisingly, this defect in metacyclogenesis was compensated in cultures that were passaged using enriched EP.1 meta rather than EP.1 stat (Figure $1 \mathrm{~F})$, which likely re-enter cell cycle faster thus outcompeting metacyclic-competent parasites. 
bioRxiv preprint doi: https://doi.org/10.1101/2021.03.22.436378; this version posted March 22, 2021. The copyright holder for this preprint (which was not certified by peer review) is the author/funder, who has granted bioRxiv a license to display the preprint in perpetuity. It is made available under aCC-BY-NC-ND 4.0 International license.

Transcriptome profiling informs on mechanisms underlying fitness tradeoff. We performed RNAseq analyses using poly(A+)-enriched mRNA obtained from three replicates of EP.1 and LP.1 log, stat, and EP.1 meta parasites. The low yield in LP.1 meta parasites precluded their analysis by RNA-seq. Principal component and hierarchical clustering analyses demonstrated that transcript profiles of EP.1 and LP.1 parasites grouped according to stage, indicating that stage-specific expression changes in $\log$, stat and meta parasites dominate over those associated with the EP.1/LP.1 promastigote fitness tradeoff (Supplementary Table 2a-f, Figure 2A, Figure S3A). Significant stage-specific changes were observed in EP.1 and LP.1 parasites during the log-stat transition for respectively $54.2 \%$ and $49.3 \%$ of the transcripts and ca. $35 \%$ of the promastigote transcriptome was modulated between EP.1 stat and EP.1 meta (Figure 2B and S3B, Supplementary Table 2a-f).

We next assessed changes in transcript abundance observed at logarithmic growth phase in LP.1 compared to EP.1 promastigotes to gain first insight into pathways associated with in vitro fitness gain (i.e. accelerated growth). We identified 345 transcripts with increased abundance in LP.1 log (Figure 2C, left panel), with functional enrichment observed for the GO terms 'ribosome biogenesis', 'ribosome assembly', and 'rRNA processing' (Figure 2C, middle panel and Supplementary Table 2g). LP.1 log fitness gain thus likely reflects an increase in translation efficiency, which may allow for accelerated growth observed in these cells. Further interrogation combining GO analysis and manual inspection of gene annotation revealed increased abundance of other transcripts implicated in various regulatory processes linked to proliferation (Figure $2 \mathrm{C}$, right panel), including epigenetic or epi-transcriptomic regulation (10 genes, e.g. Ld1S_110036500 encoding for a Pseudouridylate synthase 10, Ld1S_260334600 encoding for a RNA pseudouridylate synthase and Ld1S_330597500 encoding for a Histone methyltransferase DOT1) and cell cycle control (12 genes, e.g. Ld1S_050817000 encoding for CYC2-like cyclin, or Ld1S_330603400 encoding for the cell division control protein CDC45) (see Supplementary Table 2e for more examples).

In contrast, no GO enrichment was observed for the 437 transcripts showing reduced abundance in LP.1 log. Manual inspection of gene annotations identified various pathways implicated in metabolism and energy production (e.g. genes encoding for respiratory chain proteins, amino acid and sugar metabolism, fatty acid biosynthesis), signaling (numerous kinases and phosphatases) and flagellar biogenesis (including four genes encoding for paraflagellar rod components) (Figure S3C). These pathways suggest a potential retooling of the LP.1 log energy metabolism in the nutrition-rich culture environment, and selection against motility, which may liberate the energy required for faster growth. Surprisingly, one of the most significant decreases in transcript abundance in these cells was observed for a gene encoding for a $5 \mathrm{~S}$ ribosomal protein, along four other genes encoding for ribosomal components (Supplementary Table 2e), even though other ribosomal components 
were upregulated in LP.1 log. This result provided a first indication that LP.1 log fitness gain not only depends on the quantity, but likely also the quality or type of ribosomes, which may be adapted either for higher translation efficiency or translational control of the fitness-adapted expression profile.

Finally, we assessed changes in transcript abundance observed at stationary growth phase in LP.1 compared to EP.1 promastigotes to gain further insight into mechanisms of fitness loss (i.e. attenuated infectivity). We identified 662 transcripts with increased abundance in LP.1 stat (Figure 2D, left panel). Enrichment was observed for the GO terms 'ribosomal large subunit assembly', 'rRNA processing' and 'regulation of gene expression' (Figure 2D, middle panel). In contrast, LP.1 stat promastigotes showed reduced abundance for 710 transcripts, including several transcripts linked to the GO terms 'histone phosphorylation', 'centriole replication', 'telomere maintenance', 'reciprocal meiotic recombination' and 'response to DNA damage stimulus' (Figure 2D, right panel). Likewise, decreased abundance was observed for transcripts associated with the GO terms 'evasion or tolerance of immune response of other organism involved in symbiotic interaction', 'cellular response to osmotic stress' and 'arginine biosynthetic process' (Figure 2D, right panel). The depletion of LP.1 stat parasites in these functions could be associated with attenuated infectivity observed in LP.1 meta (see Figure 1D), which may be further aggravated by reduced expression in these parasites of 31 amastin surface glycoproteins and amastin-like proteins as well as 32 other genes previously associated with parasites infectivity, including GP63 (Supplementary Table 2f) [51, 52].

In conclusion, our data link increased fitness in in vitro growth of LP.1 log to a gain-offunction phenotype associated with proliferation, ribosomal biogenesis, and translation. Conversely, the reduced fitness in infectivity of LP.1 stat was associated with a loss-of-function phenotype linked to reduced expression of virulence genes.

Post-transcriptional adaptation during promastigote fitness gain. The observed changes in transcript abundance during in vitro fitness gain may be caused by increased gene dosage due to chromosomal amplification $[8,10]$. Indeed, comparative genomic analysis of EP.1 and LP.1 parasites revealed aneuploidy for 9 chromosomes during culture adaptation, including trisomies for chromosomes (chr) 5, 23, 26, and 33 (Figure S4A and Supplementary Table 3a). Plotting normalized genomic versus transcriptomic read depth ratios for EP.1 and LP.1 log and stat parasites correlated $75 \%$ of the up-regulated genes in LP.1 log and $42 \%$ in the LP.1 stat promastigotes with amplified chromosomes (Figure 3A), affecting various biological processes associated with the LP.1 fitness tradeoff (Figure S5). Nevertheless, interrogating more specifically the read-depth ratios for trisomic 
chr 5 and 26 uncovered surprisingly high, gene dosage-independent fluctuations of RNA abundance in EP.1 and LP.1 promastigotes (Figure 3B and Supplementary Table 4d-e). While a significant fraction of transcripts on the trisomic chromosomes showed the expected 1.5-fold increase in abundance, numerous transcripts either exceeded this increase or on the contrary were expressed at lower-thanexpected abundance. Such fluctuations were also observed for the LP.1/EP.1 ratios of disomic chromosomes (see chr 36, Figure 3B as an example and Supplementary Table 4f).

We next assessed this phenomenon at genome-wide level by normalizing the RNA-seq read counts to the corresponding DNA-seq reads. Direct comparison of the normalized transcript output in EP.1 versus LP.1 revealed a global, gene dosage-independent increase in transcript abundance in LP.1 log, as would be expected for the increased metabolism in these fast-growing cells (Figure 3C, left panel). In the absence of transcriptional regulation in Leishmania, such a global increase may indicate important changes in RNA turnover, i.e. increased mRNA stability. No difference was observed for metabolically less active, cell-cycle arrested EP.1 and LP.1 stat (Figure 3C, right panel). Post-transcriptional increase in mRNA abundance was observed in LP.1 log parasites for the biological processes 'rRNA processing', 'ribosome biogenesis', 'translational initiation', and 'nuclear transport' (Figure 3D and Supplementary Table 4g). In contrast, manual inspection revealed posttranscriptional decrease in abundance of mRNAs involved in flagellar biogenesis or EP.1-specific, ribosomal components (Figure 3E and Supplementary Table 4b). Significantly, reduction of both DNA and RNA read depth was observed for two NIMA-related protein kinases on chr 36 that we previously associated with in vitro fitness gain (Bussotti et al., in preparation) (see Figure 3E and Supplementary Table 4b), firmly linking the depletion in RNA and DNA abundance and eventually the deletion of these genes to accelerated growth.

In conclusion, the global analysis of the EP.1 and LP.1 transcriptomes uncovers posttranscriptional regulation as an important novel processes that may affect Leishmania fitness gain, which can likely buffer against deleterious effects of genome instability and adapt mRNA abundance in a gene dosage-independent manner to a given environment.

The fitness-adapted proteome is highly robust and enriched for GO terms associated with ribosomal biogenesis and post-transcriptional regulation. We applied a label-free, quantitative proteomics approach to assess how genomic and post-transcriptional adaptation during in vitro fitness gain impact protein abundance. Analyzing four independent, biological replicates of EP and LP strains (termed EP.2-5 and LP.2-5, Figure S6) identified a total of 6,050 proteins considering all samples, including 59 and 110 proteins that were exclusively detected in LP and EP parasites, 
bioRxiv preprint doi: https://doi org/10.1101/2021.03.22.436378; this version posted March 22, 2021. The copyright holder for this preprint (which was not certified by peer review) is the author/funder, who has granted bioRxiv a license to display the preprint in perpetuity. It is made available under aCC-BY-NC-ND 4.0 International license.

respectively (Figure $4 \mathrm{~A}$ and Supplementary Table 5). Considering all proteins that showed a statistically different abundance (Figure 4B), the majority of differentially expressed genes were shared in all four independent LP strains (566 of 788 total, 71\%). These data reveal a surprising convergence of the fitness-adapted proteomes despite possible karyotypic variations between strains (Figure S4B), and inform on common pathways that are under convergent selection in LP strains during in vitro fitness gain. Just as observed on RNA levels, flagellar biogenesis is clearly under negative selection during culture adaptation, with reduced protein abundance observed for 38 proteins linked to motility encoded on 20 chromosomes (Figure $4 \mathrm{C}$ and Supplementary Table $5 \mathrm{~g}$ ). Another key process associated with adaptation was translation: 30 proteins encoded on 11 different chromosomes were under positive selection in LP strains (e.g. various ribosomal proteins of the 39S, 40S, 60S, L22e, and S25 families, or the ribosomal assembly protein RRB1), while only two RNA binding proteins encoded on two chromosomes were under negative selection in the same parasites (Figure $4 \mathrm{C}$ and Supplementary Table 5c).

We next assessed the level of correlation between protein abundance, gene dosage variation and transcript abundance to gain further insight into regulatory mechanisms underlying Leishmania fitness gain. Even though the proteomics data set was obtained with four independent biological replicates (EP.2-5 and LP.2-5), the highly reproducible nature of the chr 5 and chr 26 trisomies observed in all our previous experimental evolution experiments (Figure S4B) provided a useful benchmark to assess correlations between the different data sets for at least these chromosomes. Based on our systems comparison, three different regulatory clusters can be distinguished for chr 5 and 26: Cluster 1 includes proteins whose change in abundance correlates to gene dosage and RNA abundance (Figure 4D left and right panels, upper right and lower left quadrants), including three DNA J proteins, a HSP70 like protein and BiP, suggesting that increased stress resistance may be the driving force for the selection of these aneuploidies (Supplementary Table 6h). Cluster 2 includes proteins whose levels only correlate with mRNA abundance but not gene dosage, suggesting posttranscriptional regulation (Figure 4D right panel, see also Figure $3 \mathrm{~A}$ and Supplementary Table 6i). Finally, cluster 3 represents proteins whose levels do not correlate with mRNA abundance (Figure 4D left panel, lower right and upper left quadrants), which are either regulated at translational levels or by protein turn-over (Supplementary Table 6j).

Gene ontology analysis of all proteins that fall into regulatory cluster 1 (as defined by the upper right and lower left quadrants of figure 4D) revealed a strong enrichment for the term 'posttranscriptional regulation of gene expression' (Figure 4E and Supplementary Table 6b and 6c). This enrichment is driven by the coordinated increase in expression of various proteins with known functions in RNA turnover (e.g. pumilio-domain protein encoded by Ld1S_330590200) and a series of 
proteins previously linked to post-transcriptional regulation in T. brucei such as EIF4G1 or PRP38 premRNA processing factor [53-55]. In addition, the enrichment for the GO term 'ciliary cell motility' is driven by the under-representation of this process in the LP proteome (Figure S6G and Supplementary Table 6b and 6c).

In conclusion, the Leishmania proteome undergoes reproducible, gene dosage-dependent and -independent changes during fitness gain in vitro. The robustness of proteomic adaptation indicates the presence of regulatory mechanisms that compensates for the genetic and transcriptomic variability between independent LP strains. At least under our experimental conditions, gene dosage-dependent changes modulate post-transcriptional regulation, which results in stabilization of various transcripts implicated in rRNA processing and ribosomal biogenesis. This indicates the generation of fitness-adapted ribosomes, which could generate the observed proteomic robustness. The role of ncRNAs in ribosomal biogenesis [56] primed us in the following to carry out a dedicated small RNome analysis in EP and LP parasites to further assess the generation of specialized ribosomes.

Mapping the Leishmania non-coding transcriptome correlates snoRNA expression and rRNA modification to Leishmania fitness gain. Non-coding (nc) RNAs such as small nuclear (sn), small nucleolar (sno), ribosomal ( $r$ ) or transfer ( $t$ ) RNAs play essential roles in post-transcriptional regulation and translational control $[22,57]$. While our data suggested an important role of these regulatory processes in genome-independent fitness gain (see above), they did not inform on underlying ncRNAs as our RNAseq analyses used poly $(\mathrm{A}+$ )-enriched mRNA. We therefore performed a dedicated analysis of the small RNome in EP.1 and LP.1 L. donovani parasites. We first annotated our PacBio LD1S reference genome for ncRNAs using bioinformatics approaches (ortholog mapping, de novo annotation) as well as unmapped RNAseq reads of post-ribosomal supernatants (PRS) that are enriched in ncRNAs (Figure S7). These efforts established a very first repertoire of ncRNAs in any Leishmania species and identified 1504 genes encoding for snoRNA organized in 42 clusters on twenty four chromosomes, 83 tRNA genes, 12 snRNA genes and 140 SL RNA genes (Supplementary Table1, Figure 5A and B). Considering the trisomic chromosomes, we found 269 snoRNA genes on chr 5, 22 on chr 23, 160 on chr 26, and 193 on chr 33. We investigated more specifically the role of snoRNAs in LP.1 fitness gain given the enrichment of the fitness-adapted transcriptome in the GO terms 'ribosomal biogenesis' and 'rRNA processing' (see Figure 2C). snoRNAs guide specific modifications of rRNA, such as methylation and pseudouridylation, which in turn change the specificity of the ribosome towards certain mRNAs and thus control translation [58]. We prepared 
whole cell lysates from both EP.1 log and LP.1 log parasites, removed the ribosomes by ultracentrifugation, and prepared libraries from the post-ribosomal supernatant (PRS). From 174 detected snoRNAs, 93 showed a more than 2-fold change in LP.1 compared to EP.1, revealing a global increase of snoRNA abundance during fitness gain (Supplementary Table 7). Increased abundance was confirmed for 7 out of the 8 snoRNAs probed by Northern blot analysis of the PRS (Figure $5 C$ and $D$ ).

Next, we examined if the increase in sno RNA abundance affected the level of rRNA pseudouridylation ( $\Psi$ ) by applying a modified RNAseq protocol (termed $\Psi$-seq) using total RNA from EP.1 and LP.1. We detected two hyper-modified rRNA sites in all three biological replicates at positions $\Psi 1265$ and $\Psi 1282$ inside the peptidyl transferase center (PTC), which correlated with the increased abundance of the corresponding snoRNAs that guide these modifications (Figure 5F, Supplementary Table 7) [59]. Our data thus provide a first link of snoRNA expression and rRNA modification to Leishmania fitness gain, which further supports the generation of fitness-adapted ribosomes and suggests translational control - in addition to genomic and post-transcriptional adaptation - as yet another, gene dosage-independent mechanism of Leishmania evolutionary adaptation. 


\section{Discussion}

A common hallmark of all microbial pathogens is their capacity to adapt to unpredictable fluctuations in their host environments through an evolutionary process, where genetically heterogenous individuals constantly compete for survival [60]. Here we combined experimental evolution and integrative systems approaches to uncover mechanisms of fitness gain in the human pathogen Leishmania donovani. Our study provides first evidence that these parasites combine regulatory processes at genomic, post-transcriptomic and translational levels to establish highly robust fitness phenotypes while maintaining genetic heterogeneity thereby avoiding genetic death.

In the absence of classical, promoter-driven control of gene expression, Leishmania relies on alternative mechanisms to regulate transcript and protein abundance, including regulated mRNA turn over and translational control $[5,61]$. These parasites further use a highly unusual, genomic form of gene expression regulation, where changes in chromosome and gene copy number control transcript abundance via gene dosage [7-10]. Previous studies allowed us to link these forms of genome instability to fitness gain in vitro as judged by the highly reproducible karyotypic changes observed during culture adaptation in independent clinical and animal-derived $L$. donovani isolates $[8,10,19]$. Positive selection of chromosome amplification is further sustained by the independent evolutionary experiment conducted in this study, which once more revealed amplification of chromosomes 5 and 26 as key drivers of in vitro fitness gain. Such karyotypic changes are not exclusive to culture adaptation but have been documented in L. donovani tissue amastigotes [10], and in drug resistant clinical Leishmania isolates [13]. Similar to stress-adaptation in fungi [62], karyotypic changes thus may provide the genetic diversity required for Leishmania to evolve beneficial phenotypes in response to environmental change. However, such structural mutations simultaneously affect the expression level of hundreds of genes, raising questions on the nature of the coding sequences that drive karyotypic selection during parasite adaptation, and on the mechanisms that suppress deleterious gene dosage effects while preserving beneficial ones. Applying an integrative systems approach on promastigote parasites during culture adaptation (early passage, EP and late passage, LP) allowed us to address these important open questions.

Comparative genomic, transcriptomic and proteomic analyses of EP and LP promastigote parasites revealed a gene dosage-dependent increase in mRNA and protein abundance for genes implicated in RNA turnover, including RNA-binding proteins known to regulate mRNA stability [63], pumillo domain proteins known to regulate ncRNA abundance [64], and a series of proteins that were associated with trypanosomatid mRNA-binding and post-translational regulation in recent, 
bioRxiv preprint doi: https://doi.org/10.1101/2021.03.22.436378; this version posted March 22, 2021. The copyright holder for this preprint (which was not certified by peer review) is the author/funder, who has granted bioRxiv a license to display the preprint in perpetuity. It is made available under aCC-BY-NC-ND 4.0 International license.

genome-wide functional screens [53-55]. Positive selection of chromosome amplifications during $L$. donovani culture adaptation thus is likely driven by genes that establish an adaptive, posttranscriptional interface. This interface may regulate differential mRNA stability during fitness gain, which can compensate for deleterious gene dosage effects by selective mRNA degradation, while at the same time boosting the expression of beneficial genes conferring stability to selected mRNAs. Assessment of gene-dosage-independent expression changes indeed correlated both mRNA degradation and stabilization to the observed fitness phenotype. A number of transcripts implicated in flagellar biogenesis showed reduced stability during fitness gain, which correlated with reduced mobility (data not shown). This coordinated process likely involves shared cis-regulatory sequence elements in the transcripts' 3'UTR that are recognized by the RNA-binding proteins [5]. Loss of flagellar function during $L$. donovani fitness gain has been observed in other independent evolutionary experiments (Bussotti et al., in preparation) and thus represents a reproducible, convergent phenomenon that may liberate ATP for energetically demanding processes that are under positive selection during culture adaptation. Indeed, a large number of transcripts implicated in highly energy-demanding ribosomal biogenesis and translation were stabilized in LP parasites. The differential regulation of mRNA abundance observed in our experimental evolution system thus establishes a first link of post-transcriptional regulation to Leishmania fitness gain.

In culture, fitness (defined as reproductive success of a given population) is largely synonymous to proliferation, which depends on the number of ribosomes and the cell's translational potential [65]. However, fitness-adapted mRNA turnover not only changed the quantity but also the quality of the Leishmania ribosomes as judged by the differential expression of various $40 \mathrm{~S}$ and $60 \mathrm{~S}$ ribosomal protein isoforms in EP and LP parasites (see Supplementary Tables 2 and 5). Such dynamic regulation of ribosomal biogenesis suggests the formation of specialized ribosomes, which not only may increase translation efficiency in these fast-growing LP parasites, but also control translation of unwanted mRNAs, thus providing an additional filter (next to differential RNA stability) to eliminate toxic gene dosage effects. Changes in expression and modification of different rRNA genes, ribosomal proteins, and translation factors indeed can control preferential translation of different subsets of mRNAs in other organisms [66], including stage-specific translation in Dictyostelium discoideum [67, 68], zebrafish development [69], or cancer [70]. Conducting a dedicated analysis of the L. donovani non-coding (nc) RNome, we have provided further support to the existence of such fitness-adapted ribosomes in Leishmania. First, we observed post-transcriptional upregulation of a large number of snoRNAs and five (out of a total of 9) pseudouridylate synthases in LP compared to EP promastigotes (see Supplementary Table 4). Second, these snoRNA expression changes correlated to changes in the pseudouridinylation $(\Psi)$ profile of the rRNA peptidyl transferase center (PTC) that catalyzes peptide 
bond formation and peptide release [71]. Similar $\Psi$ hyper-modification of rRNA was previously observed in bloodstream form trypanosomes and likely contributes to stage-specific adaptation [21]. Given the high coding density of chr 5 and chr 26 for snoRNAs and the functional enrichment of these chromosomes for GO term 'rRNA processing' (see Figure S4C), it is interesting to speculate that their convergent amplification in all our evolution experiments may be driven by their ncRNA content and their requirement for fitness-adapted translation. Indeed, defects in rRNA pseudouridylation affect ribosomal ligand binding and translational fidelity in eukaryotic cells [72], and changes in PTC modification were shown to affect both the ribosome structure and activity in yeast [73]. The combination of (i) different rRNA isoforms, (ii) hundreds of snoRNAs and differentially modified rRNA sites, (iii) diverse $40 \mathrm{~S}$ and $60 \mathrm{~S}$ ribosomal proteins, and (iv) the formation of different translation complexes [74-76] defines a vast ribosomal landscape in Leishmania. Translational control via fitnessadapted ribosomes likely fine-tunes expression and provides proteomic and phenotypic robustness to adapting parasite populations, which thus can maintain genetic diversity and evolvability despite constant natural selection [10].

In conclusion, our data uncover Leishmania evolutionary adaptation as an emergent property of a highly complex process that integrates variations in gene dosage with correlating changes in transcript abundance for genes implicated in post-transcriptional regulation and the establishment of specialized ribosomes capable to compensate for toxic gene dosage effects (see Figure 5G). Even though our results are largely correlative in nature, our model is supported by the convergence of the genomic, transcriptomic and proteomic signals we observed between independent populations, which thus are the result of natural selection rather than random genetic drift. Our findings challenge the current genome-centric approach to Leishmania epidemiology and identify the Leishmania noncoding RNome as well as regulatory circuits at transcriptional and translational levels as potential novel sources for biomarker discovery. Finally, our model may be of relevance to other pathogenic systems that gain fitness through genome instability, including fungal infection and cancer.

\section{Acknowledgements}

This work was supported by the Fondation de la Recherche Médicale contract FDT201805005619 (LP), the Agence Nationale pour la Recherche Labex 'Integrative Biology of Emerging Infectious Diseases' contract ANR-10-LABX-62-IBEID and Labex 'French Alliance for Parasitology and Health Care' contract ANR-11-LABX-0024 (GFS, LP, PP, GB), the Campus France Franco-Israeli Programme 
Hubert Curien Maimonide 2018 (GFS and SM), the seeding grant from the Institut Pasteur International Department to the LeiSHield Consortium and the EU H2O2O project LeiSHield-MATI REP-778298-1 (GB), the France Génomique National infrastructure, funded as part of the " Investissements d'Avenir " program managed by the Agence Nationale pour la Recherche contract ANR-10-INBS-09 (HV, RL, CP), and the ERD Funds, project CePaViP (CZ.02.1.01/0.0/0.0/ 16_019/0000759) (BV, JS, PV). We thank the CEA-CNRGH for its contribution to the sequencing costs and all the CEA-CNRGH staff who contributed to sample preparation and sequencing for their excellent technical assistance. 


\section{References}

1. WHO, Leishmaniasis in high-burden countries: an epidemiological update based on data reported in 2014, in Wkly Epidemiol Rec. 2016. p. 287-96.

2. Lindgren, E., et al., Public health. Monitoring EU emerging infectious disease risk due to climate change. Science, 2012. 336(6080): p. 418-9.

3. Koonin, E.V. and Y.I. Wolf, Evolution of microbes and viruses: a paradigm shift in evolutionary biology? Front Cell Infect Microbiol, 2012. 2: p. 119.

4. Michaeli, S., Trans-splicing in trypanosomes: machinery and its impact on the parasite transcriptome. Future Microbiol, 2011. 6(4): p. 459-74.

5. Clayton, C.E., Gene expression in Kinetoplastids. Curr Opin Microbiol, 2016. 32: p. 46-51.

6. Sterkers, Y. and E.L. Walton, The Leishmania chromosome lottery. Microbes Infect, 2014. 16(1): p. 2-5.

7. Rogers, M.B., et al., Chromosome and gene copy number variation allow major structural change between species and strains of Leishmania. Genome Res, 2011. 21(12): p. 2129-42.

8. Dumetz, F., et al., Modulation of Aneuploidy in Leishmania donovani during Adaptation to Different In Vitro and In Vivo Environments and Its Impact on Gene Expression. mBio, 2017. 8(3).

9. Iantorno, S.A., et al., Gene Expression in Leishmania Is Regulated Predominantly by Gene Dosage. mBio, 2017. 8(5).

10. Prieto Barja, P., et al., Haplotype selection as an adaptive mechanism in the protozoan pathogen Leishmania donovani. Nat Ecol Evol, 2017. 1(12): p. 1961-1969.

11. Brotherton, M.-C., et al., Proteomic and Genomic Analyses of Antimony Resistant Leishmania infantum Mutant. PLOS ONE, 2013. 8(11): p. e81899.

12. Downing, T., et al., Whole genome sequencing of multiple Leishmania donovani clinical isolates provides insights into population structure and mechanisms of drug resistance. Genome Res, 2011. 21(12): p. 2143-56.

13. Laffitte, M.N., et al., Plasticity of the Leishmania genome leading to gene copy number variations and drug resistance. F1000Res, 2016. 5: p. 2350.

14. Leprohon, P., et al., Gene expression modulation is associated with gene amplification, supernumerary chromosomes and chromosome loss in antimony-resistant Leishmania infantum. Nucleic Acids Res, 2009. 37(5): p. 1387-99.

15. Ubeda, J.M., et al., Genome-wide stochastic adaptive DNA amplification at direct and inverted DNA repeats in the parasite Leishmania. PLoS Biol, 2014. 12(5): p. e1001868.

16. Zhang, W.W., et al., Genetic analysis of Leishmania donovani tropism using a naturally attenuated cutaneous strain. PLoS Pathog, 2014. 10(7): p. e1004244.

17. Yona, A.H., et al., Chromosomal duplication is a transient evolutionary solution to stress. Proc Natl Acad Sci U S A, 2012. 109(51): p. 21010-5.

18. Negrini, S., V.G. Gorgoulis, and T.D. Halazonetis, Genomic instability--an evolving hallmark of cancer. Nat Rev Mol Cell Biol, 2010. 11(3): p. 220-8.

19. Bussotti, G., et al., Leishmania Genome Dynamics during Environmental Adaptation Reveal Strain-Specific Differences in Gene Copy Number Variation, Karyotype Instability, and Telomeric Amplification. mBio, 2018. 9(6).

20. Bakin, A. and J. Ofengand, Mapping of the 13 pseudouridine residues in Saccharomyces cerevisiae small subunit ribosomal RNA to nucleotide resolution. Nucleic Acids Res, 1995. 23(16): p. 3290-4.

21. Chikne, V., et al., A pseudouridylation switch in rRNA is implicated in ribosome function during the life cycle of Trypanosoma brucei. Sci Rep, 2016. 6: p. 25296.

22. Rajan, K.S., et al., Pseudouridines on Trypanosoma brucei spliceosomal small nuclear RNAs and their implication for RNA and protein interactions. Nucleic Acids Res, 2019. 47(14): p. 7633-7647. 
23. Liang, X.H., Y.X. Xu, and S. Michaeli, The spliced leader-associated RNA is a trypanosomespecific $\operatorname{sn}(0)$ RNA that has the potential to guide pseudouridine formation on the SL RNA. RNA, 2002. 8(2): p. 237-46.

24. Li, H., et al., The Sequence Alignment/Map format and SAMtools. Bioinformatics, 2009. 25(16): p. 2078-9.

25. DePristo, M.A., et al., A framework for variation discovery and genotyping using nextgeneration DNA sequencing data. Nat Genet, 2011. 43(5): p. 491-8.

26. Quinlan, A.R. and I.M. Hall, BEDTools: a flexible suite of utilities for comparing genomic features. Bioinformatics, 2010. 26(6): p. 841-2.

27. Liao, Y., G.K. Smyth, and W. Shi, featureCounts: an efficient general purpose program for assigning sequence reads to genomic features. Bioinformatics, 2014. 30(7): p. 923-30.

28. Cokelaer, T., et al., Sequana': a Set of Snakemake NGS pipelines. Journal of Open Source Software, 2017. 2(16): p. 352.

29. Martin, M., Cutadapt removes adapter sequences from high-throughput sequencing reads. 2011, 2011. 17(1): p. 3.

30. Dobin, A., et al., STAR: ultrafast universal RNA-seq aligner. Bioinformatics, 2012. 29(1): p. 1521.

31. Team, R.C., R: A Language and Environment for Statistical Computing, in R Foundation for Statistical Computing. 2016.

32. Love, M.I., W. Huber, and S. Anders, Moderated estimation of fold change and dispersion for RNA-seq data with DESeq2. Genome Biol, 2014. 15(12): p. 550.

33. Benjamini, Y. and Y. Hochberg, Controlling the False Discovery Rate: A Practical and Powerful Approach to Multiple Testing. Journal of the Royal Statistical Society. Series B (Methodological), 1995. 57(1): p. 289-300.

34. Cox, J. and M. Mann, MaxQuant enables high peptide identification rates, individualized p.p.b.-range mass accuracies and proteome-wide protein quantification. Nat Biotechnol, 2008. 26(12): p. 1367-72.

35. Cox, J., et al., Andromeda: a peptide search engine integrated into the MaxQuant environment. J Proteome Res, 2011. 10(4): p. 1794-805.

36. Cox, J., et al., Accurate proteome-wide label-free quantification by delayed normalization and maximal peptide ratio extraction, termed MaxLFQ. Mol Cell Proteomics, 2014. 13(9): p. 251326.

37. Perez-Riverol, Y., et al., The PRIDE database and related tools and resources in 2019: improving support for quantification data. Nucleic Acids Res, 2019. 47(D1): p. D442-D450.

38. Wieczorek, S., et al., DAPAR \& ProStaR: software to perform statistical analyses in quantitative discovery proteomics. Bioinformatics, 2017. 33(1): p. 135-136.

39. Smyth, G.K., limma: Linear Models for Microarray Data, in Bioinformatics and Computational Biology Solutions Using R and Bioconductor, R. Gentleman, et al., Editors. 2005, Springer New York: New York, NY. p. 397-420.

40. Ritchie, M.E., et al., limma powers differential expression analyses for RNA-sequencing and microarray studies. Nucleic Acids Res, 2015. 43(7): p. e47.

41. Giai Gianetto, Q., et al., Calibration plot for proteomics: A graphical tool to visually check the assumptions underlying FDR control in quantitative experiments. Proteomics, 2016. 16(1): p. 29-32.

42. Pounds, S. and C. Cheng, Robust estimation of the false discovery rate. Bioinformatics, 2006. 22(16): p. 1979-87.

43. Aulner, N., et al., High content analysis of primary macrophages hosting proliferating Leishmania amastigotes: application to anti-leishmanial drug discovery. PLoS Negl Trop Dis, 2013. 7(4): p. e2154.

44. Volf, P. and V. Volfova, Establishment and maintenance of sand fly colonies. J Vector Ecol, 2011. 36 Suppl 1: p. S1-9. 
45. Seblova, V., et al., Phlebotomus orientalis sand flies from two geographically distant Ethiopian localities: biology, genetic analyses and susceptibility to Leishmania donovani. PLoS Negl Trop Dis, 2013. 7(4): p. e2187.

46. Sadlova, J., et al., The stage-regulated HASPB and SHERP proteins are essential for differentiation of the protozoan parasite Leishmania major in its sand fly vector, Phlebotomus papatasi. Cell Microbiol, 2010. 12(12): p. 1765-79.

47. Pescher, P., et al., Quantitative proteome profiling informs on phenotypic traits that adapt Leishmania donovani for axenic and intracellular proliferation. Cell Microbiol, 2011. 13(7): p. 978-91.

48. Spath, G.F. and S.M. Beverley, A lipophosphoglycan-independent method for isolation of infective Leishmania metacyclic promastigotes by density gradient centrifugation. Exp Parasitol, 2001. 99(2): p. 97-103.

49. Rogers, M.E., M.L. Chance, and P.A. Bates, The role of promastigote secretory gel in the origin and transmission of the infective stage of Leishmania mexicana by the sandfly Lutzomyia longipalpis. Parasitology, 2002. 124(Pt 5): p. 495-507.

50. Lei, S.M., N.M. Romine, and J.K. Beetham, Population changes in Leishmania chagasi promastigote developmental stages due to serial passage. J Parasitol, 2010. 96(6): p. 1134-8.

51. McGwire, B. and K.P. Chang, Genetic rescue of surface metalloproteinase (gp63)-deficiency in Leishmania amazonensis variants increases their infection of macrophages at the early phase. Mol Biochem Parasitol, 1994. 66(2): p. 345-7.

52. Spath, G.F., et al., Lipophosphoglycan is a virulence factor distinct from related glycoconjugates in the protozoan parasite Leishmania major. Proc Natl Acad Sci U S A, 2000. 97(16): p. 9258-63.

53. Erben, E.D., et al., A genome-wide tethering screen reveals novel potential posttranscriptional regulators in Trypanosoma brucei. PLoS Pathog, 2014. 10(6): p. e1004178.

54. Lueong, S., et al., Gene expression regulatory networks in Trypanosoma brucei: insights into the role of the mRNA-binding proteome. Mol Microbiol, 2016. 100(3): p. 457-71.

55. de Pablos, L.M., et al., The mRNA-bound Proteome of Leishmania mexicana: Novel Genetic Insight into an Ancient Parasite. Mol Cell Proteomics, 2019. 18(7): p. 1271-1284.

56. Rajan, K.S., et al., Unique Aspects of rRNA Biogenesis in Trypanosomatids. Trends Parasitol, 2019. 35(10): p. 778-794.

57. Kamina, A.D. and N. Williams, Ribosome Assembly in Trypanosomatids: A Novel Therapeutic Target. Trends Parasitol, 2017. 33(4): p. 256-257.

58. Watkins, N.J. and M.T. Bohnsack, The box C/D and H/ACA snoRNPs: key players in the modification, processing and the dynamic folding of ribosomal RNA. Wiley Interdiscip Rev RNA, 2012. 3(3): p. 397-414.

59. Eliaz, D., et al., Genome-wide analysis of small nucleolar RNAs of Leishmania major reveals a rich repertoire of RNAs involved in modification and processing of rRNA. RNA Biol, 2015. 12(11): p. 1222-55.

60. Lenski, R.E., What is adaptation by natural selection? Perspectives of an experimental microbiologist. PLoS Genet, 2017. 13(4): p. e1006668.

61. Clayton, C. and M. Shapira, Post-transcriptional regulation of gene expression in trypanosomes and leishmanias. Mol Biochem Parasitol, 2007. 156(2): p. 93-101.

62. Tsai, H.J. and A. Nelliat, A Double-Edged Sword: Aneuploidy is a Prevalent Strategy in Fungal Adaptation. Genes (Basel), 2019. 10(10).

63. Haile, S. and B. Papadopoulou, Developmental regulation of gene expression in trypanosomatid parasitic protozoa. Curr Opin Microbiol, 2007. 10(6): p. 569-77.

64. Goldstrohm, A.C., T.M.T. Hall, and K.M. McKenney, Post-transcriptional Regulatory Functions of Mammalian Pumilio Proteins. Trends Genet, 2018. 34(12): p. 972-990.

65. Thomas, G., An encore for ribosome biogenesis in the control of cell proliferation. Nat Cell Biol, 2000. 2(5): p. E71-2. 
66. Guo, H., Specialized ribosomes and the control of translation. Biochem Soc Trans, 2018. 46(4): p. 855-869.

67. Ramagopal, S. and H.L. Ennis, Regulation of synthesis of cell-specific ribosomal proteins during differentiation of Dictyostelium discoideum. Proc Natl Acad Sci U S A, 1981. 78(5): p. 3083-7.

68. Ramagopal, S., Induction of cell-specific ribosomal proteins in aggregation-competent nonmorphogenetic Dictyostelium discoideum. Biochem Cell Biol, 1990. 68(11): p. 1281-7.

69. Locati, M.D., et al., Expression of distinct maternal and somatic 5.8S, 185, and 285 rRNA types during zebrafish development. RNA, 2017. 23(8): p. 1188-1199.

70. Belin, S., et al., Dysregulation of ribosome biogenesis and translational capacity is associated with tumor progression of human breast cancer cells. PLoS One, 2009. 4(9): p. e7147.

71. Polacek, N. and A.S. Mankin, The ribosomal peptidyl transferase center: structure, function, evolution, inhibition. Crit Rev Biochem Mol Biol, 2005. 40(5): p. 285-311.

72. Jack, K., et al., rRNA pseudouridylation defects affect ribosomal ligand binding and translational fidelity from yeast to human cells. Mol Cell, 2011. 44(4): p. 660-6.

73. King, T.H., et al., Ribosome structure and activity are altered in cells lacking snoRNPs that form pseudouridines in the peptidyl transferase center. Mol Cell, 2003. 11(2): p. 425-35.

74. Meleppattu, S., et al., The elF3 complex of Leishmania-subunit composition and mode of recruitment to different cap-binding complexes. Nucleic Acids Res, 2015. 43(13): p. 6222-35.

75. Yoffe, Y., et al., Evolutionary changes in the Leishmania elF4F complex involve variations in the elF4E-elF4G interactions. Nucleic Acids Res, 2009. 37(10): p. 3243-53.

76. Yoffe, Y., et al., Binding specificities and potential roles of isoforms of eukaryotic initiation factor 4E in Leishmania. Eukaryot Cell, 2006. 5(12): p. 1969-79. 


\section{Figure legends}

Figure 1: Phenotypic analysis of EP.1 and LP.1 parasites reveals fitness tradeoff during culture adaptation.

(A) Histogram plot representing the generation time of EP.1 and LP.1 promastigotes in culture calculated following parasite density during logarithmic growth phase. The mean value of three independent experiments $+/$ - SD is represented, ${ }^{*} p$-value $\leq 0.05$. (B) Macrophage infection assay. The mean relative number of intracellular EP.1 (open circles) and LP.1 (grey circles) parasites +/- SD of three independent experiments using promastigotes from day-3 stationary culture is represented, ${ }^{* *}$ p-value $\leq 0.01$. (C) Histogram plot representing the percentage of EP.1 and LP.1 metacyclic forms that were enriched by Ficoll density gradient centrifugation from cultures at stationary growth phase. Each bar represents the mean $+/-$ SD of four independent experiments, $* p$-value $\leq 0.05$. (D) Macrophage infection assay using Ficoll-enriched metacyclic parasites. Percentage of infected macrophages (left panel), mean relative number of intracellular EP.1 and LP.1 parasites (middle panel) and mean number of parasites per 100 macrophages (right panel) are shown. Open triangles, EP.1 meta; close triangles, LP.1 meta. The mean values +/- SD of one triplicate experiment are shown, $* *$ p-value $\leq 0.01$. (E) Morphological characterization of EP.1 and LP.1 Ficoll-enriched metacyclic parasites. The ratio flagellum-to-body length was computed from two biological replicate experiments and the median values +/- SD are represented by the box plot with the upper and lower quartiles indicated, ${ }^{* *} p$-value $\leq 0.01$. (F) Percentage of metacyclic-like parasites recovered by Ficoll gradient centrifugation from cultures seeded successively for 6 in vitro passages with either EP.1 from stationary growth phase (stat-stat) or EP.1 metacyclic-enriched parasites (meta-meta). Mean values of two independent experiments are shown with +/-SD denoted by the bars.

Figure 2: RNA-seq analyses of EP.1 and LP.1 promastigotes reveal stage-specific changes in RNA abundance and RNA signatures of fitness gain and fitness cost.

(A) Cluster analysis of differentially expressed genes observed in triplicate RNAseq analyses of EP.1 log and LP.1 log, EP.1 stat and LP.1 stat, and EP.1 meta parasites. (B) Ratio plots of normalized RNAseq reads for EP.1 log compared to EP.1 stat (upper panel) and LP.1 log compared to LP.1 stat (lower panel). Blue and dark cyan dots represent gene expression changes with FC $>1.5$ and adjusted $p$-value $\leq 0.01$; black dots correspond to gene expression changes with adjusted $p$-value $>0.01$. Only genes with at least 10 reads in one of the two conditions were considered. Top panel, 1499 and 1501 transcripts more abundant in EP.1 log (dark cyan) and EP.1 stat (blue), respectively. Lower panel 1129 and 1384 transcripts more abundant in LP.1 log (dark cyan) and LP.1 stat (blue), respectively. (C) Differential expression profiling of LP.1 log and EP.1 log parasites. Volcano plot representing the 
changes in transcript abundances of LP.1 log and EP.1 log parasites with 345 transcripts more abundant in LP.1 log versus 437 transcripts more abundant in EP.1 log (left panel). Transcripts with significant increased abundance FC $>1.5$ and adjusted p-value $\leq 0.01$ in LP.1 log and EP.1 log are indicated respectively in cyan and blue. GO analysis of these transcripts for the category 'biological process' is shown in the middle panel and expressed as 'cluster efficiency', representing the percentage of genes associated with a given $\mathrm{GO}$ term compared to the total number of genes with annotation in any given list of genes. GO enrichment analysis of transcripts showing increased abundance in LP.1 log is shown in the right panel and expressed as 'number of genes' for the indicated gene families. (D) Differential expression profiling of LP.1 stat and EP.1 stat parasites. Volcano plot representing the changes in transcript abundances of LP.1 stat and EP.1 stat parasites with 662 transcripts more abundant in LP.1 stat versus 710 transcripts more abundant in EP.1 stat (left panel). Transcripts with significant increased abundance FC $>1.5$ and adjusted $p$-value $\leq 0.01$ in LP.1 stat and EP.1 stat are indicated respectively in cyan and blue. Results of GO analyses for the category 'biological process' performed on transcripts showing increased abundance in LP.1 stat (middle panel) and EP.1 stat (right panel) are shown.

Figure 3: RNA abundance during fitness gain is regulated by gene dosage and post-transcriptional mechanisms.

(A) Ratios of DNA and RNA normalized read counts for all genes were plotted for LP.1 log compared to EP.1 log (left panel) and for LP.1 stat compared to EP.1 stat (right panel). Green dots correspond to genes encoded on trisomic chromosomes in LP.1 parasites. The regression line is represented by the dotted red line. Pearson correlation coefficients and p-values were estimated for both ratio plots using SigmaPlot software. For LP.1 log compared to EP.1 log, $\rho=0.341, p$-value $<10^{-10}$ and for LP.1 stat compared to EP.1 stat, $\rho=0.333, p$-value $<10^{-10}$ both indicative of a medium degree of correlation. (B) Normalized coverage based on the ratio of DNA read counts in LP.1 versus EP.1 for the trisomic chromosomes 5 (upper panel) and 26 (middle panel), and the disomic chromosome 36 (lower panel). The coverage ratio is indicated by the line, while ORFs are indicated by the vertical lines. The color code reflects the DNA strand on which the ORFs are encoded. (C) Post-transcriptional regulation of transcript abundance. RNA read counts were normalized by DNA read counts and plotted for all genes in LP.1 log compared to EP.1 log (left panel) and EP.1 stat compared to LP.1 stat (right panel). Green dots correspond to genes encoded on trisomic chromosomes in LP.1. The calculated (red) and expected (blue) regression lines are represented. (D) Cluster efficiency computed from GO term-enrichment analysis for the 'biological process' category for gene dosageindependent genes. (E) Table listing selected gene dosage-dependent and -independent expression 
changes. The fold change values computed from RNA (grey bars) and DNA (black bars) normalized read counts for LP.1 versus EP.1 log parasites are shown.

Figure 4: Quantitative analysis of the fitness-adapted proteome.

(A) Volcano plot representing changes in protein abundance in EP log (blue dots, mean values of EP.2, EP.3, EP.4 and EP.5 are shown) compared to LP log (green dots, mean values of LP.2, LP.3, LP.4 and LP.5 are shown). Proteins identified by at least two peptides in at least three out of four biological replicates were considered. Colored dots indicate values with FDR $<0.01$ and fold changes $\geq 2$. The grey dots indicate non-significant expression changes. The bars indicate unique protein identifications in LP (LP only, green) and EP (EP only, blue) samples, with relative abundance indicated by the $\mathrm{iBAQ}$ value. (B) Venn diagram showing the number of proteins quantified and associated to a $p$-value $<0.01$ with increased (left panel) or decreased (right panel) abundance in all four LP log biological replicates. (C) Manual Gene ontology analysis of the proteins shared in all four LP log biological replicates expressed as 'number of genes' for the indicated gene families. (D) Double ratio plots comparing the fold changes computed for each gene between LP and EP log parasites for RNA ( $x$-axis) versus Protein (y-axis) (left panel) and DNA ( $x$-axis) versus Protein ( $y$-axis) (right panel). All proteins with LFQ values were considered to determine the protein ratio LP/EP. Grey dots represent all proteins and red dots those encoded on trisomic chromosomes 5 and 26 . Cluster 1 (Cl 1) includes proteins whose change in abundance correlates to gene dosage and RNA abundance. The regression line is represented by the dotted red line. The Pearson correlation coefficients and the $p$ values were estimated for both ratio plots using SigmaPlot software. For protein versus RNA ratio plot, $\rho=0.349$ and $p$-value $<10^{-10}$ and for protein versus DNA ratio plot, $\rho=0.145$ and $p$-value $<10^{-10}$ indicative of medium and low degree of correlation respectively. (E) GO-enrichment analysis for the category 'biological process' for proteins whose DNA and protein LP/EP ratios correlate (cluster 1, figure 4D right panel). Only proteins quantified in all four biological replicates for each condition and associated with a p-value $<0.01$ were considered for the $\mathrm{GO}$ analysis. $(\mathrm{F})$ Table listing selected genes associated with the GO term 'post-transcriptional regulation of gene expression' from the GO enrichment analysis presented in (E). Their respective fold change values computed from Protein LFQ intensities (grey bars) and DNA normalized read counts (black bars) for LP versus EP log parasites are represented. * proteins exclusive to EP log parasites.

Figure 5: The fitness tradeoff in LP promastigotes correlates with snoRNA expression changes and increased rRNA pseudouridinylation levels.

(A) Genomic map of L. donovani Ld1S ncRNA genes. (B) Composition of the small RNome identified in EP parasites. (C) Northern blot analysis of selected snoRNAs, Ld14Cs1H3 was used as loading control. 
One representative northern blot out of three is presented. (D) Histogram plot representing the fold changes between LP (red bars) and EP (blue bars) log parasites corresponding to densitometric analysis of the signals shown in (C). (E) Representative line graph of the fold change in rRNA pseudouridinylation level $(\Psi-\mathrm{fc}, \log 2)$ is presented for EP (blue line) and LP (red line). The positions where the $\Psi$ level is increased in three replicates are indicated in red. (F) The location of $\Psi$ sites in the rRNA is depicted on the secondary structure. Hypermodified sites are highlighted in red squares. The snoRNAs guiding each $\psi$ are indicated. The color code for each $\psi$ site is indicative of the organism where it was already reported. (G) Model of Leishmania evolutionary adaptation. Different environments (E1, E2) select for different fitness traits (F1, F2), which modify the parasite population structure (pop 1, pop 2). In the absence of transcriptional regulation, Leishmania exploits genome instability to generate changes in gene dosage via chromosome and gene copy number variations. These changes are either correlated (blue arrows) or not (green arrow) to changes in transcript and protein abundance. The gene dosage-regulated transcriptome and proteome (right panel) is highly enriched for the GO term 'post-transcriptional regulation of gene expression' and thus likely regulates gene dosage-independent changes in RNA abundance (red arrow, left panel). The enrichment of these transcripts in ncRNAs in turn can control RNA stability and translatability by guiding modifications of mRNA or rRNAs. This allows for (i) compensation of deleterious gene dosage effects, (ii) phenotypic robustness despite genetic heterogeneity, and (iii) maintenance of evolvability despite selection pressure. 


\section{Supplementary Figure legends}

\section{Figure S1: Macrophage infection studies.}

(A) Comparison of EP.1 stat (open circle) and LP.1 stat (grey circles) infectivity. Mean number of parasites per 100 macrophages +/-SD (left panel) and the percentage of infection (right panel) from three biological replicates are shown. (B) Comparison of EP.1 stat (open circle) and EP.1 meta (open triangle) infectivity. Mean number +/-SD of parasites per 100 macrophages (left panel), the percentage of infection (middle panel) and the relative number of intracellular parasites (right panel) from a representative experiment out of three replicates are shown. ${ }^{*}$ indicates $p$-value $\leq 0.05$.

\section{Figure S2: Morphological analysis of EP and LP metacyclic-enriched parasite fractions.}

(A) Micrographs of representative EP.1 metacyclic isolated from the sand fly thoracic part (left image), Ficoll-enriched EP.1 (middle image) and LP.1 metacyclic-like parasites (right image) from stationary culture. (B) Quantitative morphological analysis of stationary-phase and metacyclicenriched parasite populations. The box plots show the median values and the upper and lower quartiles for body length (left panel), body width (middle panel) and flagellum length (right panel). (C) Distribution of the indicated promastigote forms in EP.1 and LP.1 Ficoll-enriched metacyclic fractions.

Figure S3: Transcriptomic analysis of EP.1 and LP.1 parasites.

(A) Principal component analysis of EP.1 and LP.1 parasites from logarithmic (log) and stationary (stat) phase cultures, and after metacyclic enrichment (meta). (B) Ratio plots of normalized RNA abundance for EP.1 stat compared to EP.1 meta. Dark blue and dark cyan dots represent respectively gene expression changes with $\mathrm{FC}>1.5$ and adjusted $p$-value $\leq 0.01$; black dots correspond to gene expression changes with adjusted p-value $>0.01$. (C) Number of genes showing decreased transcript abundance in LP.1 log for the indicated GO terms.

Figure S4: Comparative genome analysis of EP and LP parasites from independent evolutionary experiments.

(A) Chromosome somy levels of EP.1 and LP.1 promastigotes. Chromosome read-depth distributions are shown in boxplots depicting the median and the upper and lower quartiles. (B) Violin plot computed from three independent evolutionary experiments representing the somy score 
distribution for each chromosome. In red are highlighted chr 5 and 26 that are trisomic in all three experiments (LP.1, LP.6 and LP.7). (C) Enrichment analysis of the trisomic chromosomes for the GO categories 'molecular function' (chr 1) and 'biological process' (5, 12, 23, 26 and 33). The bars correspond to the cluster efficiency computed from GO term-enrichment analyses.

\section{Figure S5: GO analysis of gene dosage-dependent and -independent changes in RNA abundance.}

(A, B) Enrichment analysis for the GO category 'biological process' for genes showing no changes (A) or a decrease (B) in RNA read counts after normalization to DNA read counts in LP.1 log parasites. The bars correspond to the cluster efficiency computed from the GO term enrichment analysis (BiNGO).

Figure S6: Quantitative proteomics analysis.

(A) Box plots representing the median ratio and the upper and lower quartiles of the LFQ intensity values for all LP biological replicates (LP.2, LP.3, LP.4 and LP.5) compared to the median of all EP replicates (EP.2, EP.3, EP.4 and EP.5). (B) Cluster analysis of all EP and LP samples (Ward method). (C) Ratio plot representing the mean LFQ intensity value between EP and LP for each individual, quantified protein. The experimental and the expected regression lines are shown in red and blue respectively. (D, E) Cluster efficiency for the GO category 'biological process' for proteins whose abundance correlates with increased (D) or decreased normalized DNA read counts (E) in LP log parasites. (F) Cluster efficiency for genes showing gene dosage-independent changes in protein abundance. (G) Table listing selected genes associated with the GO term 'ciliary cell motility' from the GO enrichment analysis presented in Figure 4E. Their respective fold change values computed from Protein LFQ intensities (grey bars) and DNA normalized read counts (black bars) for LP versus EP log parasites are represented.

Figure 57: Enrichment of small RNAs obtained from post-ribosomal supernatants (PRS) of EP promastigotes. $2 \times 10^{9}$ cells were disrupted by nitrogen cavitation under low salt concentration (150 $\mathrm{mM} \mathrm{KCL}$ ) in the presence of high $\mathrm{MgCl}_{2}(10 \mathrm{mM})$ followed by ribosome extraction using high $\mathrm{KCL}$ (300 $\mathrm{mM})$. The ribosomes were removed by centrifugation at $35,000 \mathrm{rpm}$ for $2 \mathrm{~h} .2 \mu \mathrm{g}$ of RNA from total lysate (Total RNA) and PRS sample were separated on a $10 \%$ polyacrylamide gel and stained with ethidium bromide. 


\section{Supplementary Tables*}

Supplementary Table 1. Transcriptomic read counts of EP and LP RNAseq analyses.

Supplementary Table 2. EP versus LP comparative transcript profiling.

Supplementary Table 2. EP versus LP comparative transcript profiling.

Supplementary Table 3. Genomic analyses of EP and LP parasites.

Supplementary Table 4. Analysis of gene copy number-independent changes in RNA abundance.

Supplementary Table 5. Proteomic analysis of EP and LP log parasites.

Supplementary Table 6. Correlation between protein abundance, gene dosage variation and transcript abundance.

Supplementary Table 7. snoRNA expression level in EP and LP parasites.

*Due to the volume of the supplementary Tables, their submission to bioRxiv as pdf files was deemed unfeasible. All supplementary Tables can be requested from the corresponding authors. 

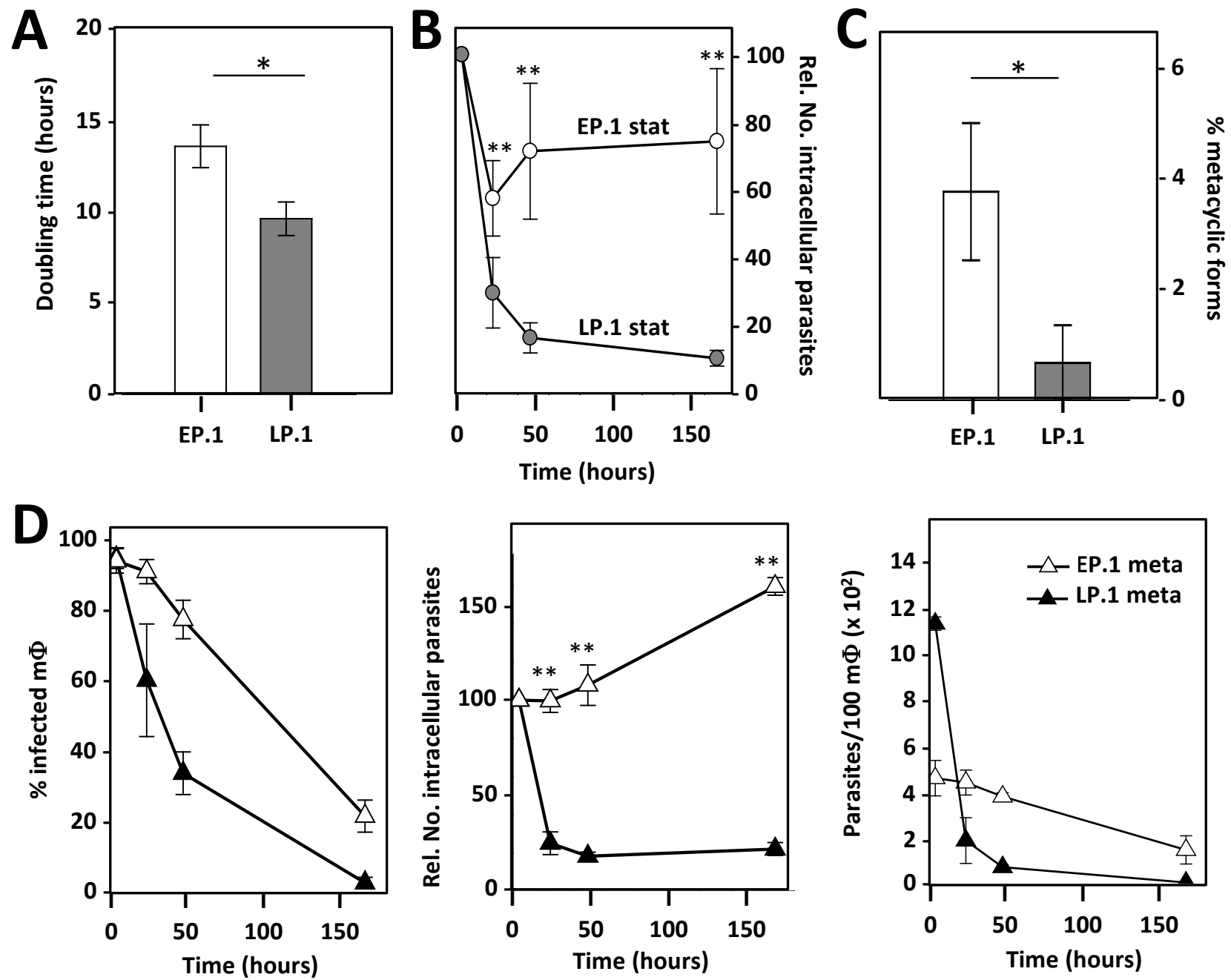

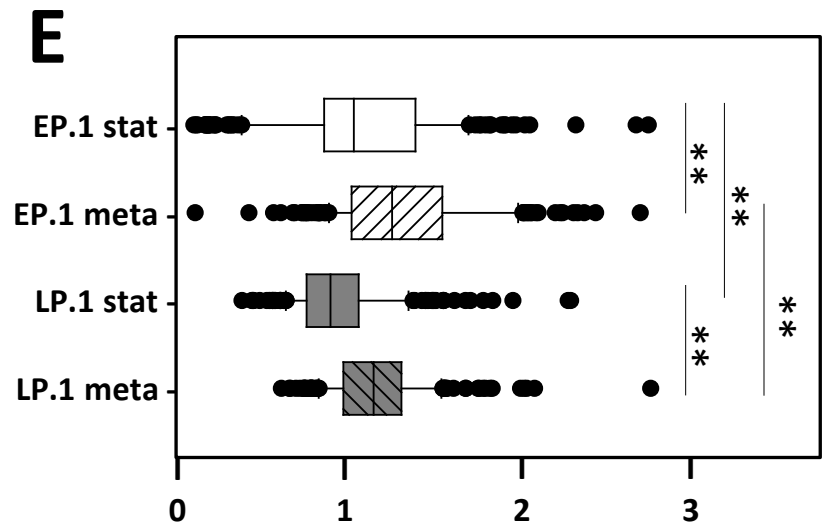

Ratio flagellum/body length

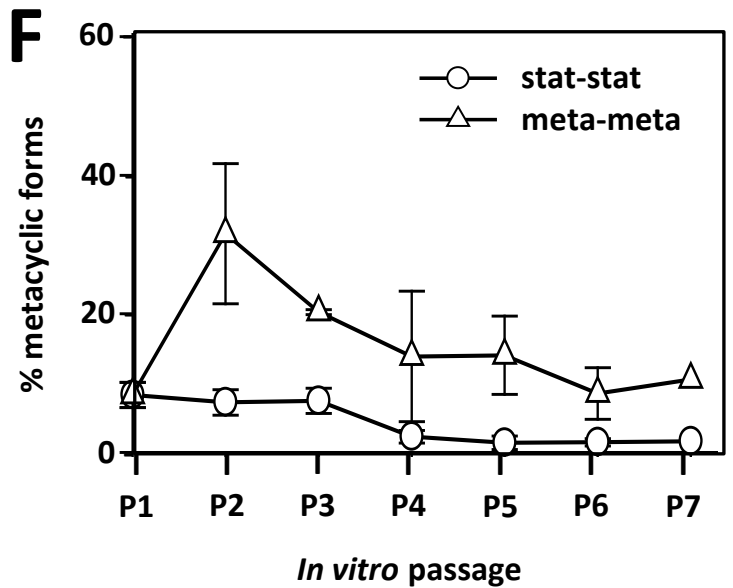

Figure 1 

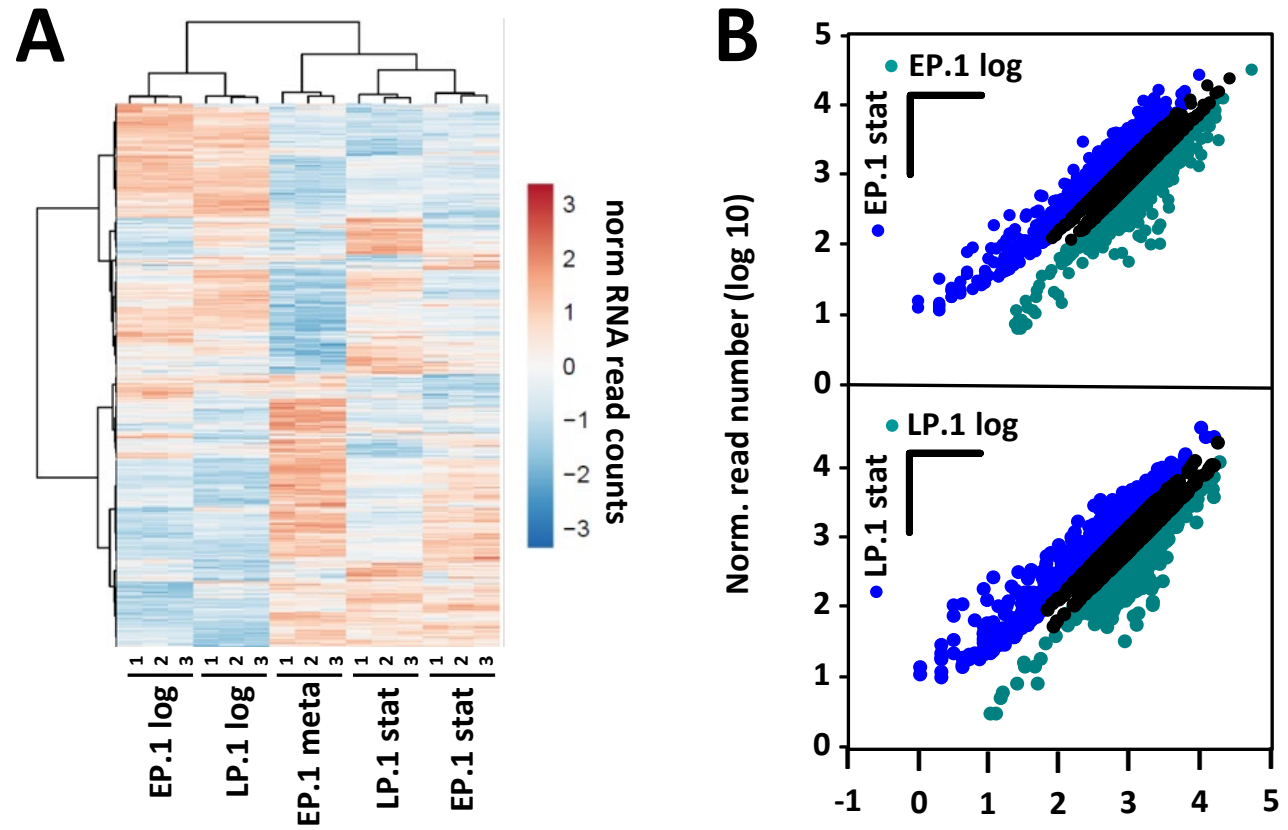

Norm. read number (log 10)

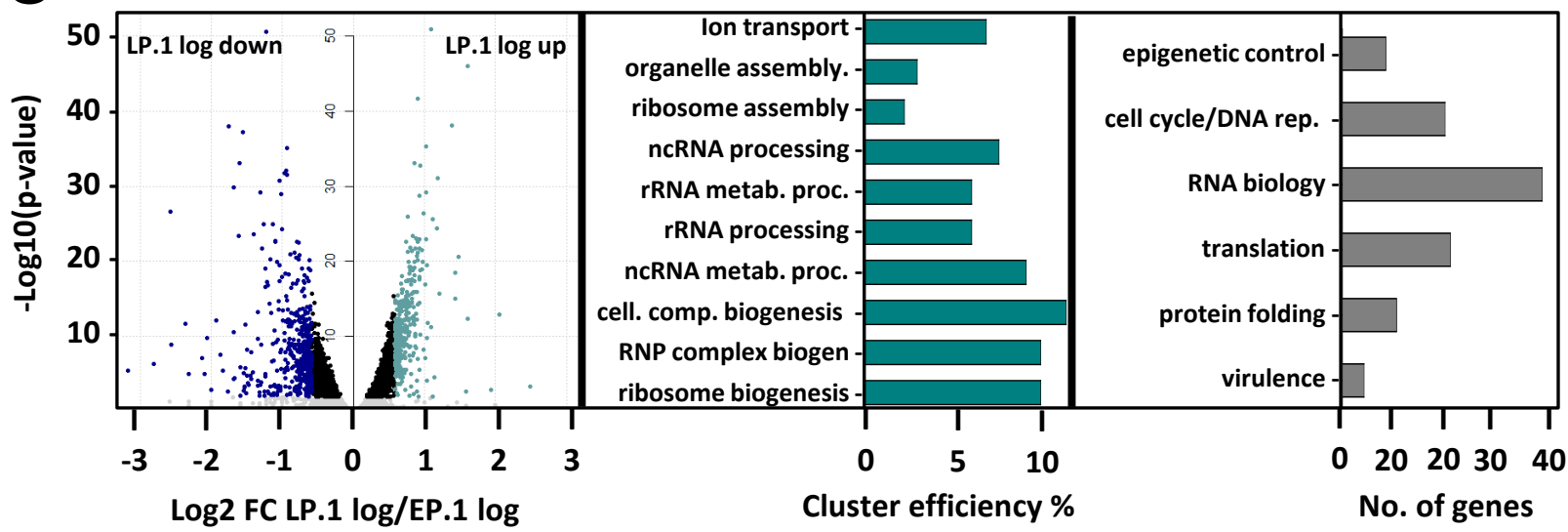

D

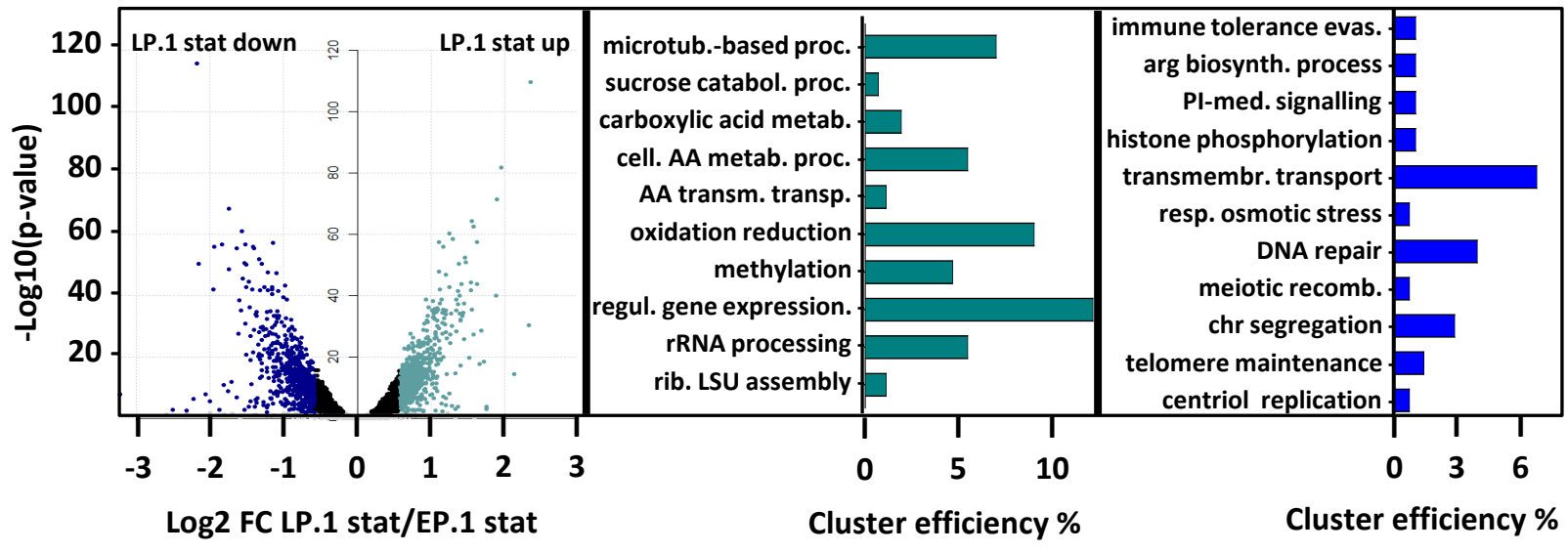


A
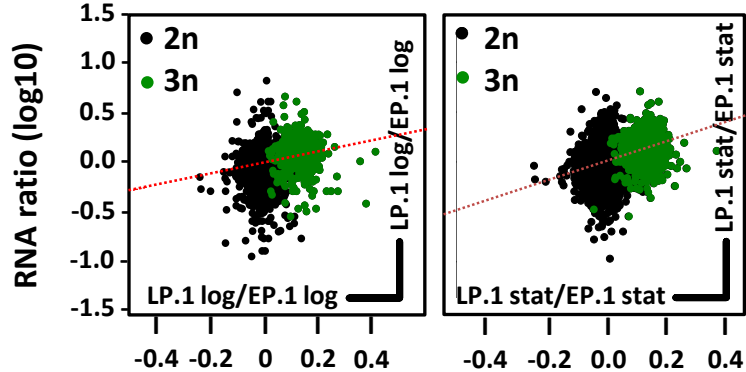

DNA ratio $(\log 10)$

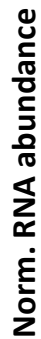
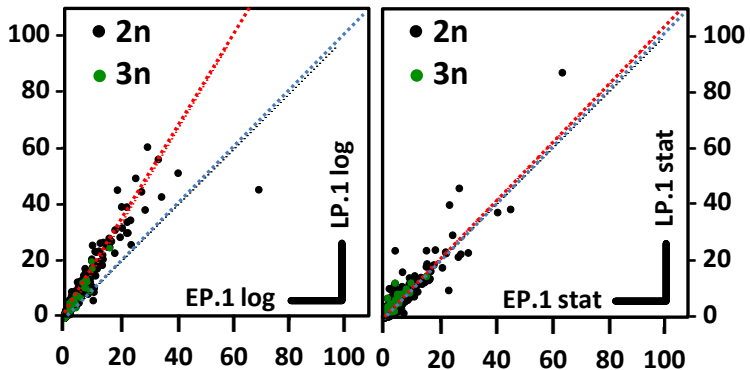

B

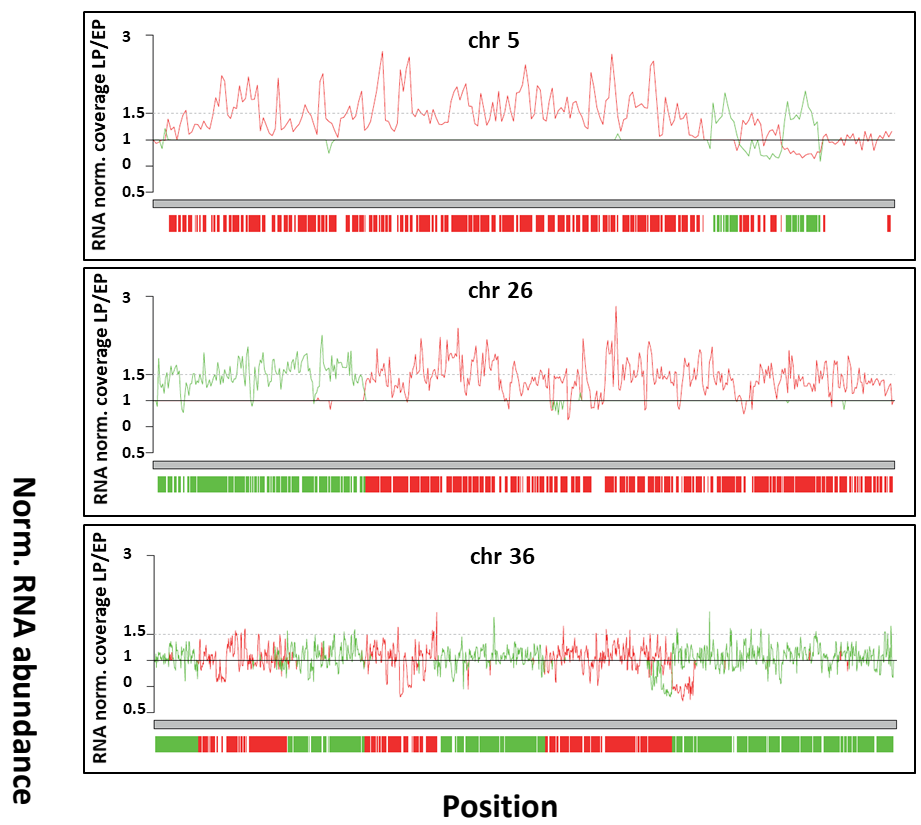

Norm. RNA abundance

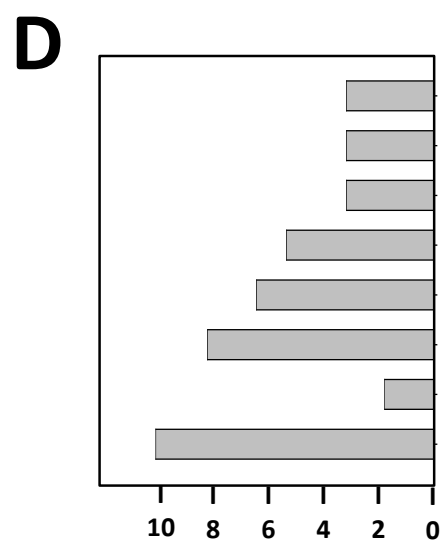

translational

initiation

nucleocytoplasmic

transport

nuclear transport

RNA modification

rRNA processing

ncRNA processing

ribosomal large

subunit biogenesis

ribosome biogenesis

Cluster efficiency (\%)

\begin{tabular}{|c|c|c|c|}
\hline Regulation & Gene id & Annotation & Fold change LP/EP \\
\hline \multirow{3}{*}{$\begin{array}{c}\text { Gene dosage } \\
\text { dependent }\end{array}$} & Ld1S_360735900 & engulfment and cell motility domain 2 , putative & $\square_{\mathrm{DNA}}$ \\
\hline & Ld1S_360735700 & serine/threonine protein kinase, putative & \\
\hline & Ld1S_360735800 & serine/threonine protein kinase, putative & \\
\hline \multirow{6}{*}{$\begin{array}{l}\text { Gene dosage } \\
\text { independent }\end{array}$} & Ld1S_360750700 & paraflagellar rod component Par4. Function unknown & \\
\hline & Ld1S_090887500 & paraflagellar rod component, putative & \\
\hline & Ld1S_360771700 & paraflagellar rod component, putative & \\
\hline & Ld1S_320549600 & paraflagellar Rod Proteome Component 9, putative & 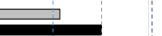 \\
\hline & Ld1S_330583300_pseudogene & paraflagellar rod component, putative & 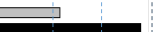 \\
\hline & Ld1S_360766000 & paraflagellar rod component, putative & $\square$ \\
\hline
\end{tabular}


LP only

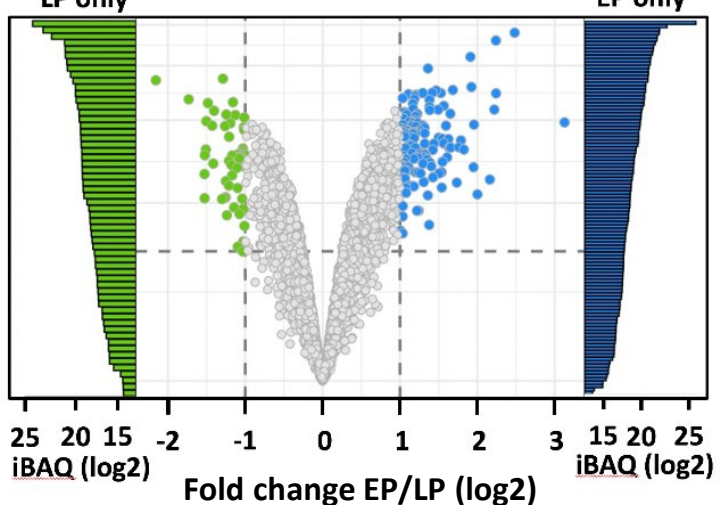

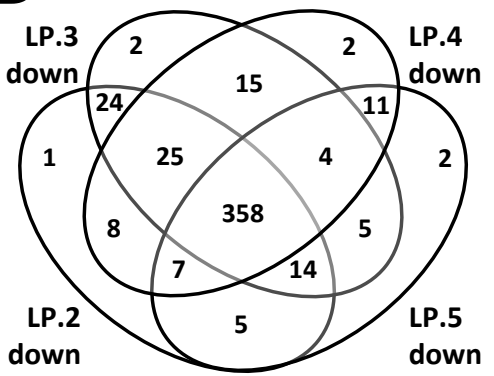

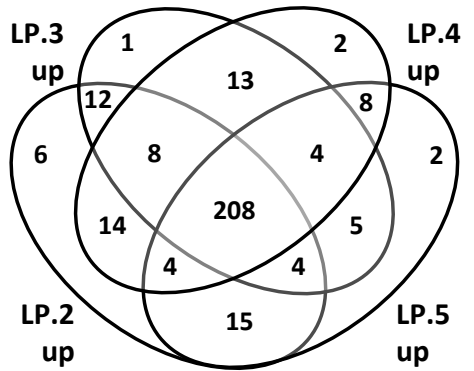

up
D

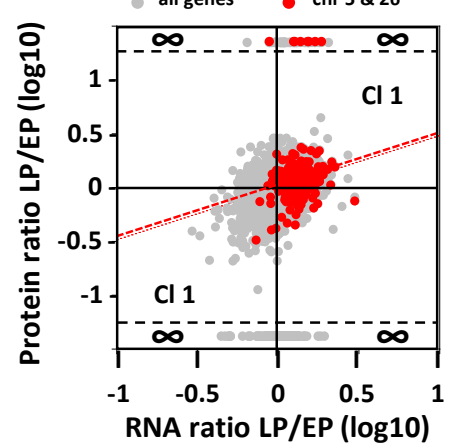

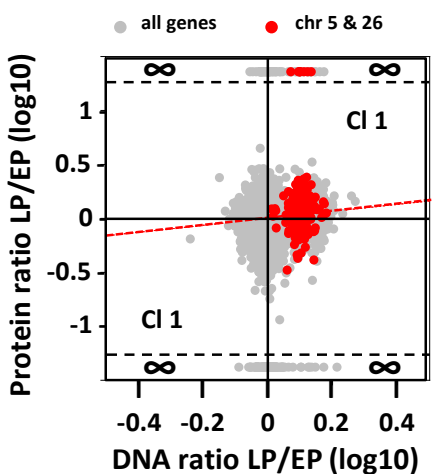
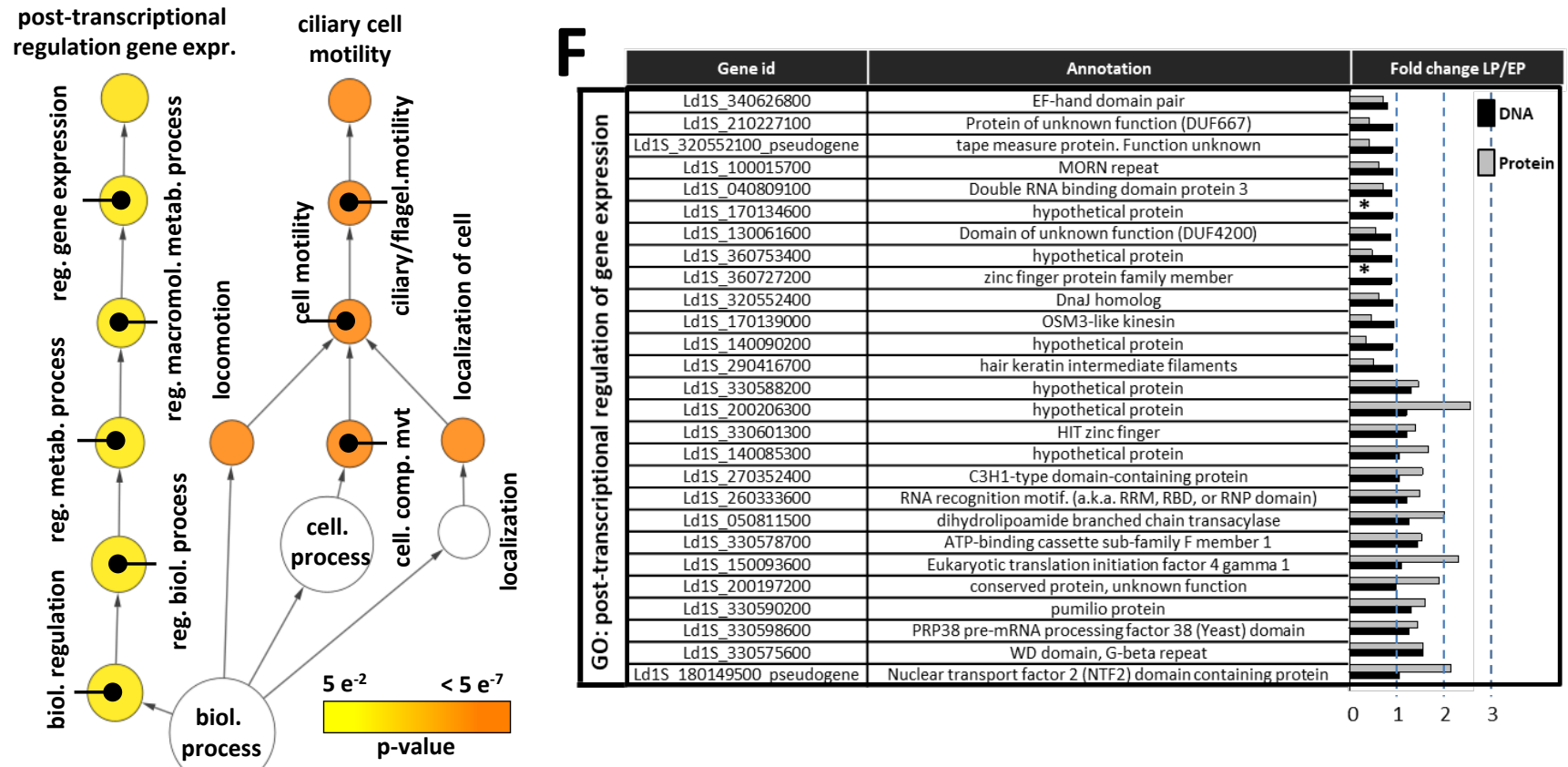

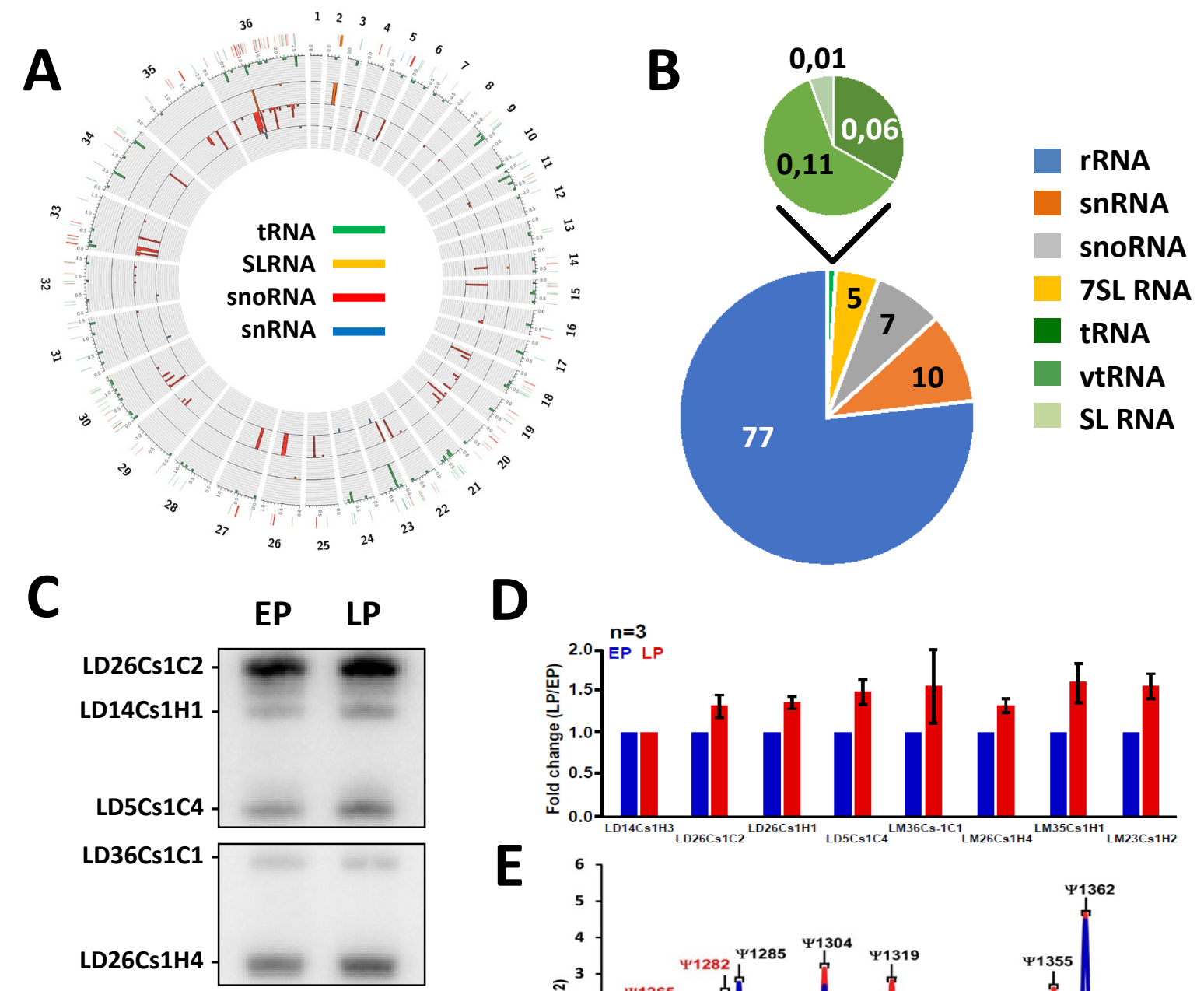

D
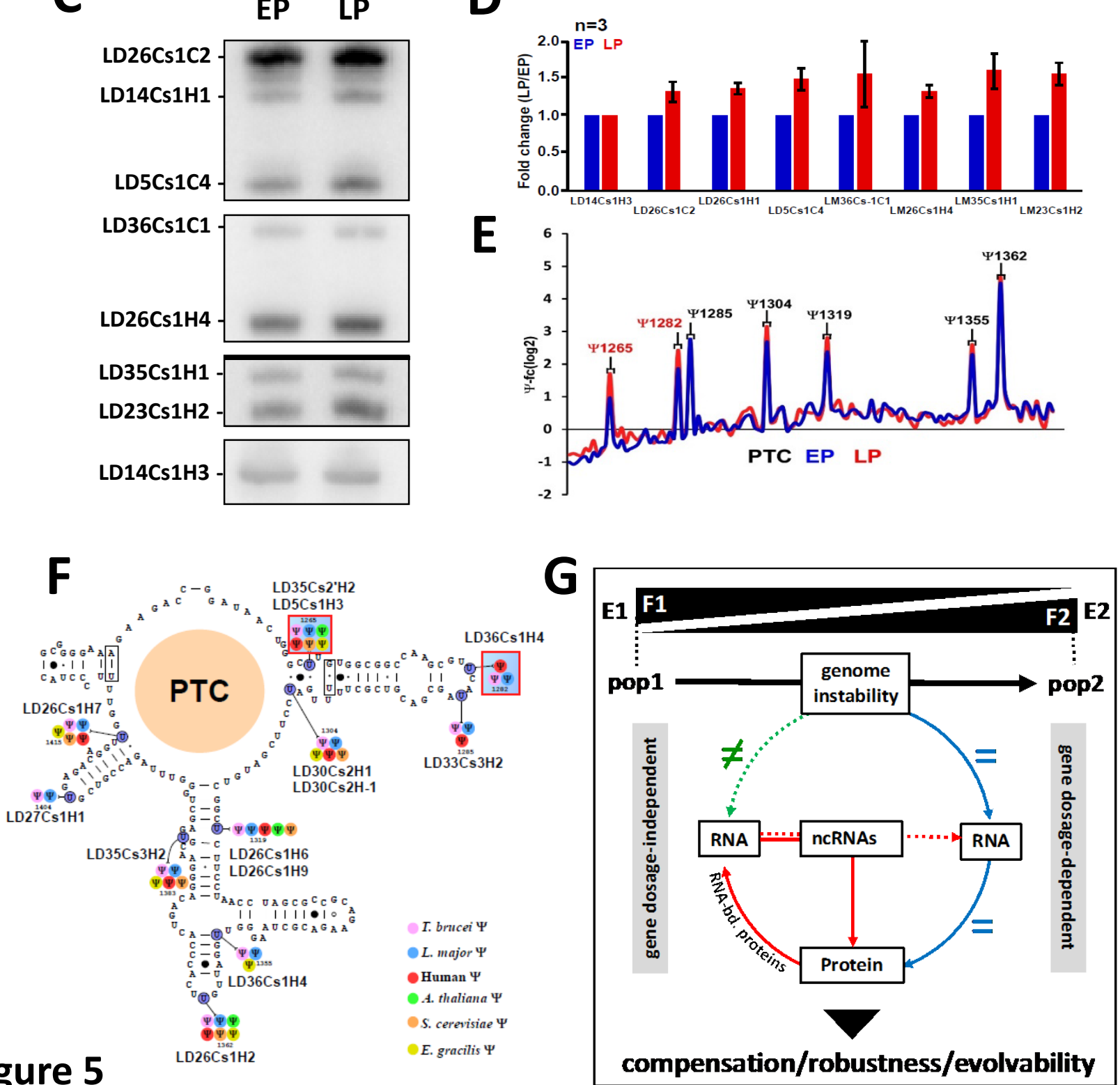

Figure 5 
Supplementary Figures 
A

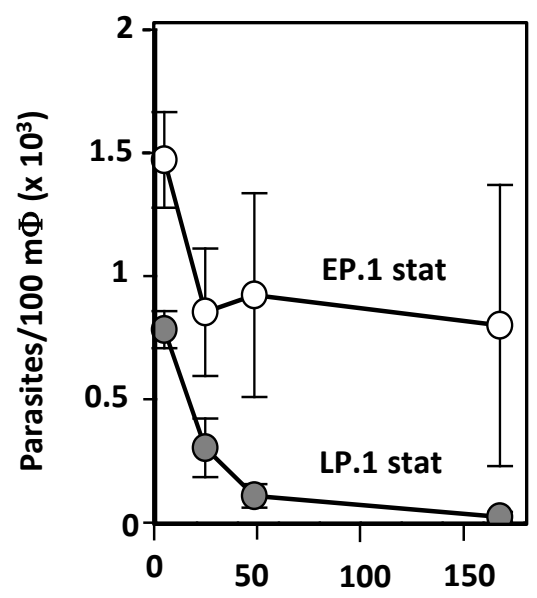

B

Time (hours)

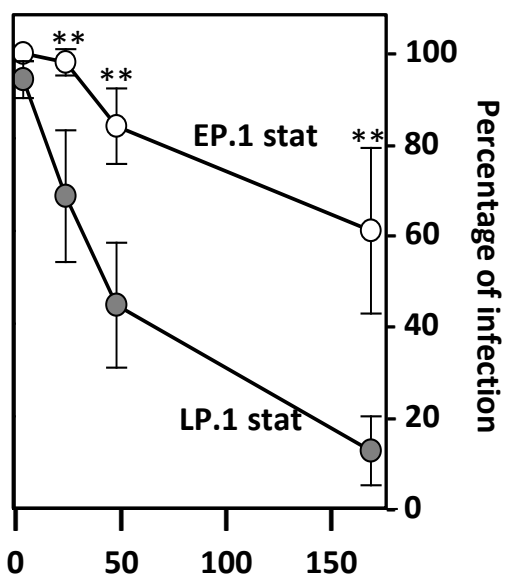

Time (hours)
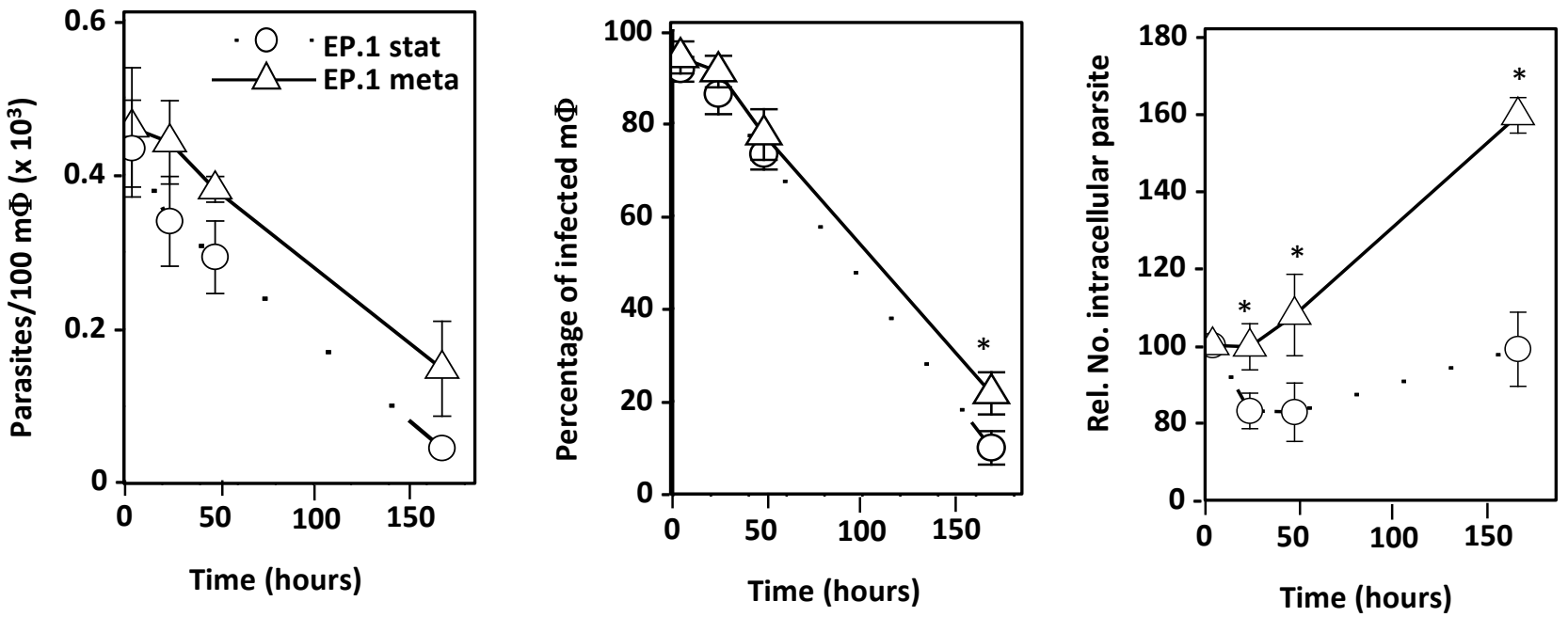
A

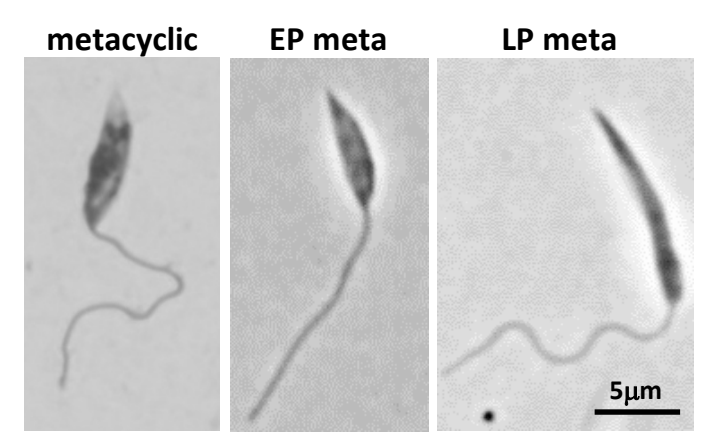

B
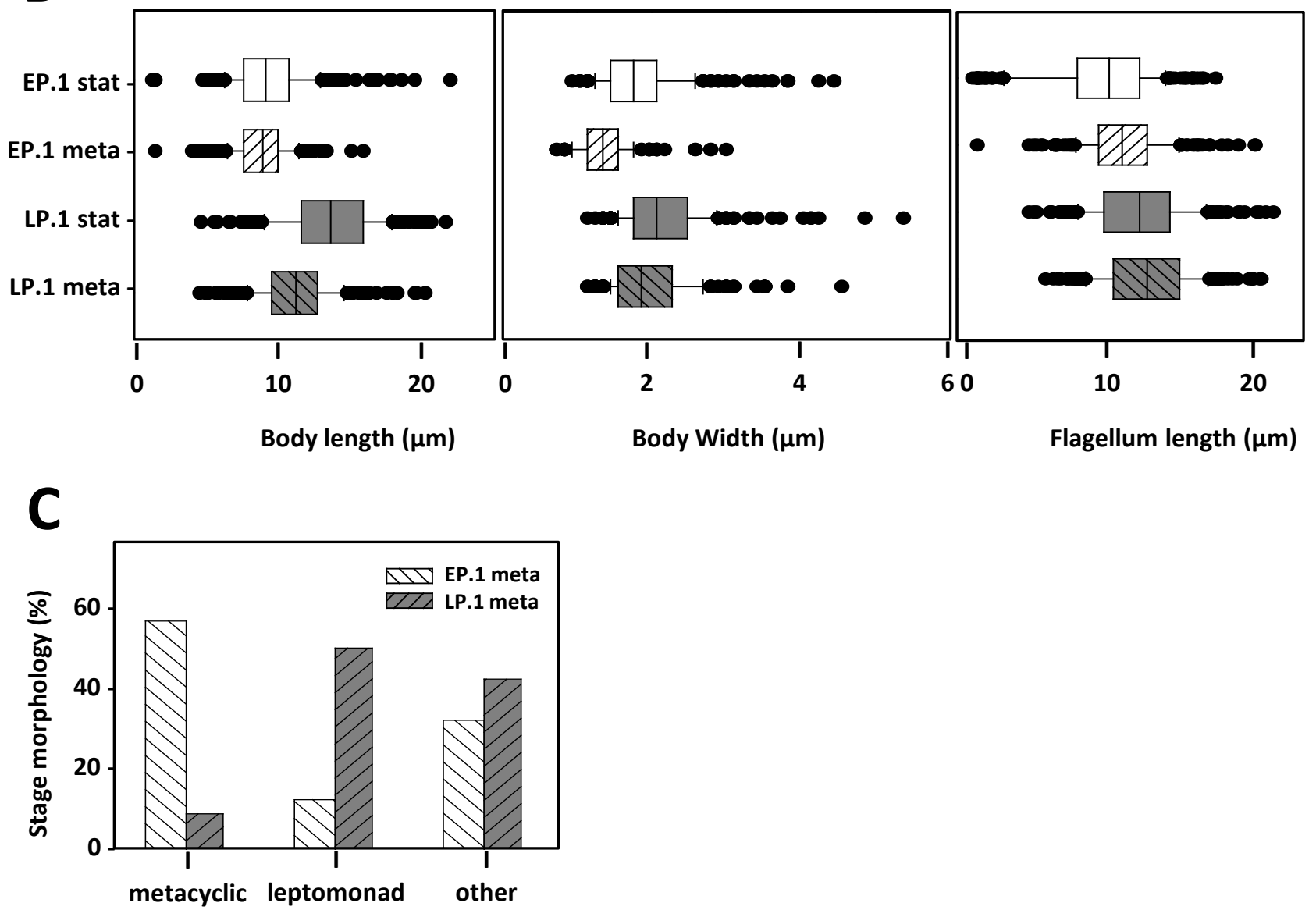

Figure S2 
A

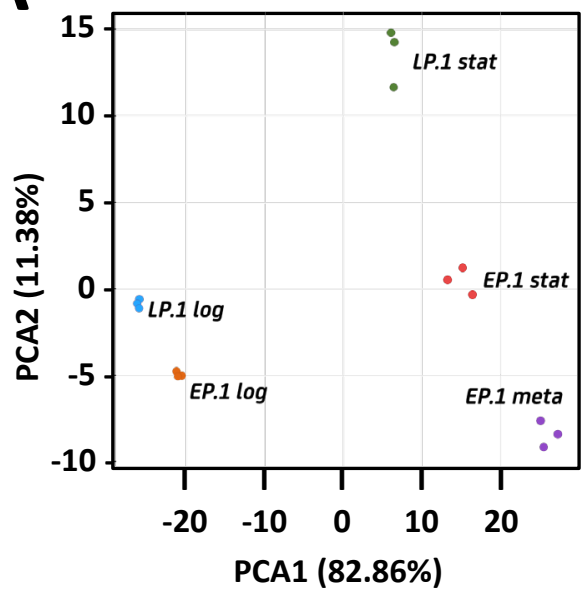

B

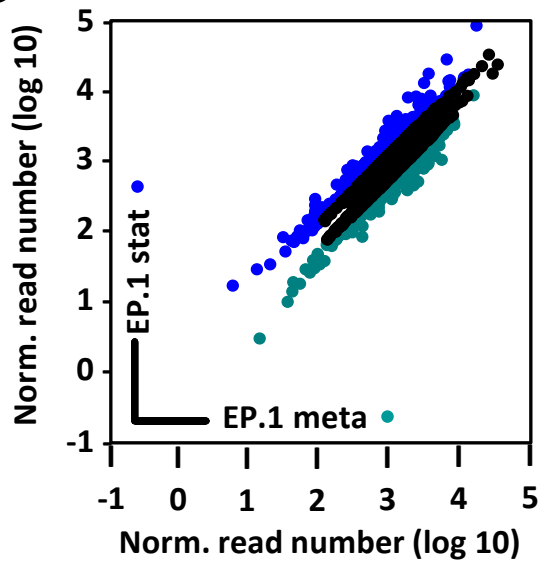

C

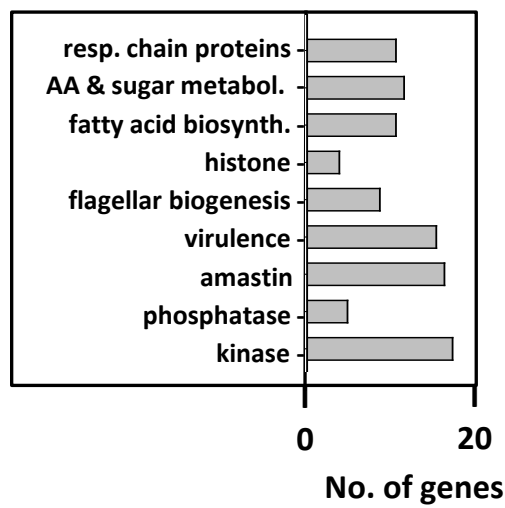


A

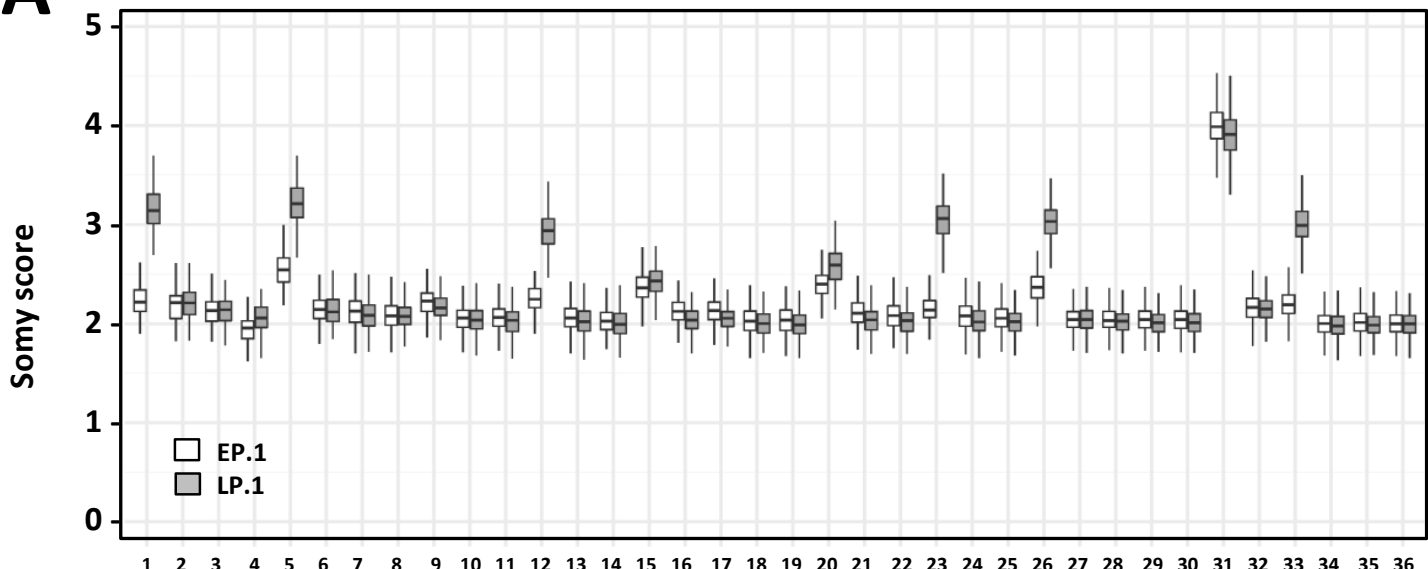

B

\section{Chromosome}

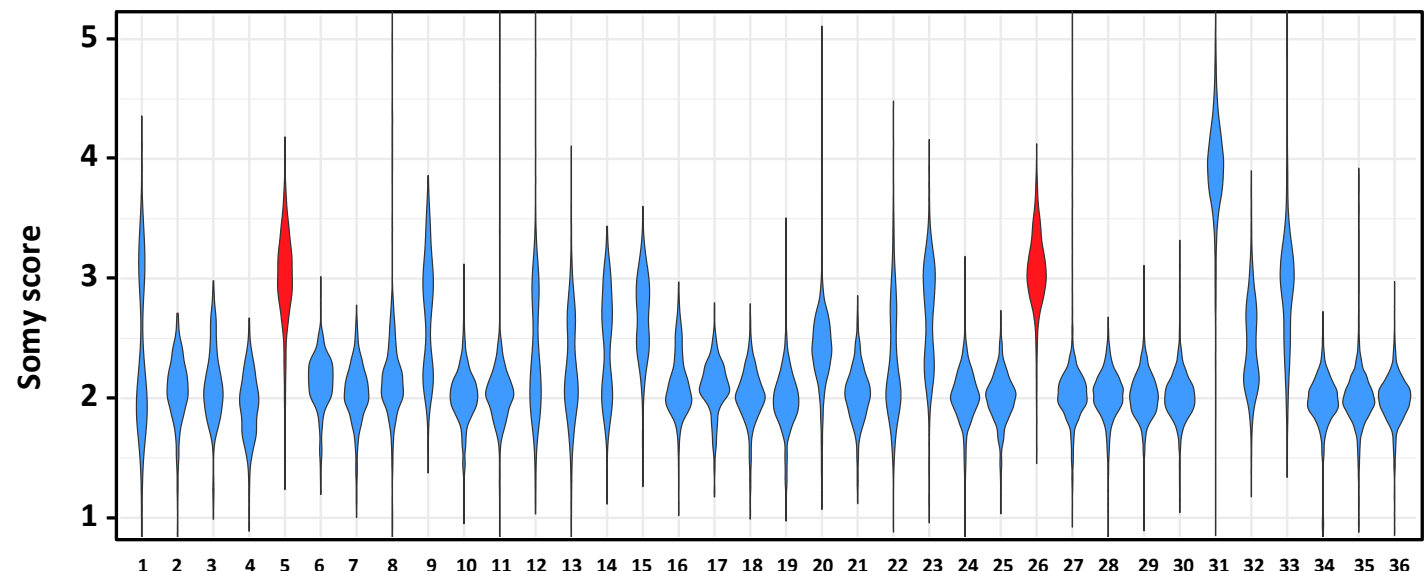

Chromosome

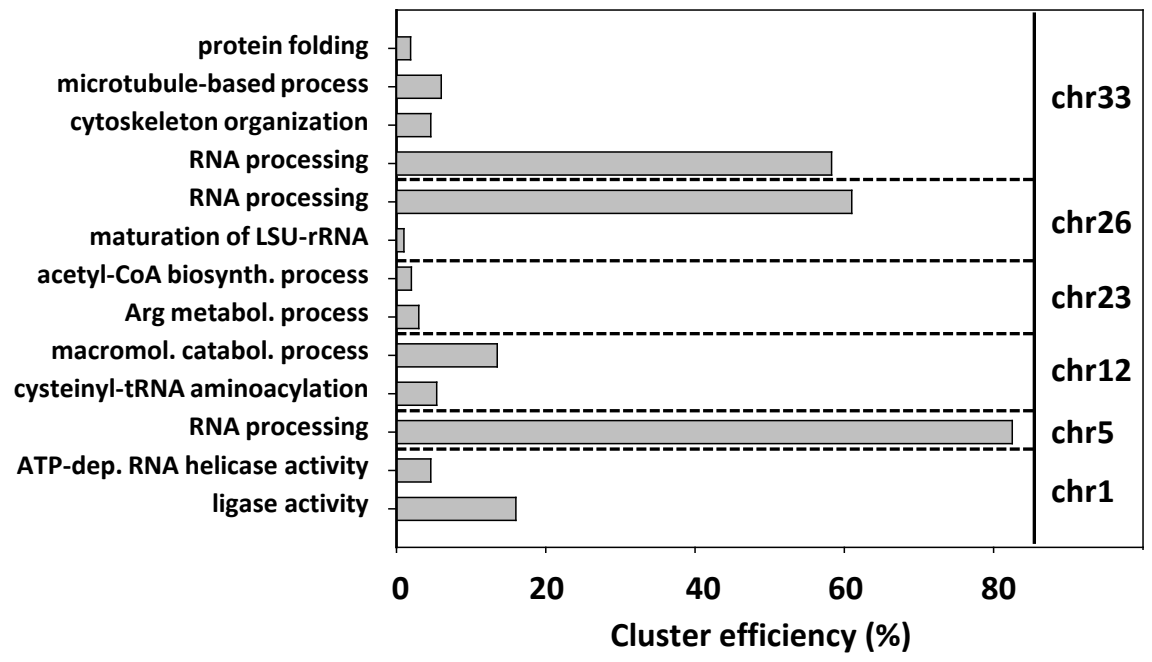



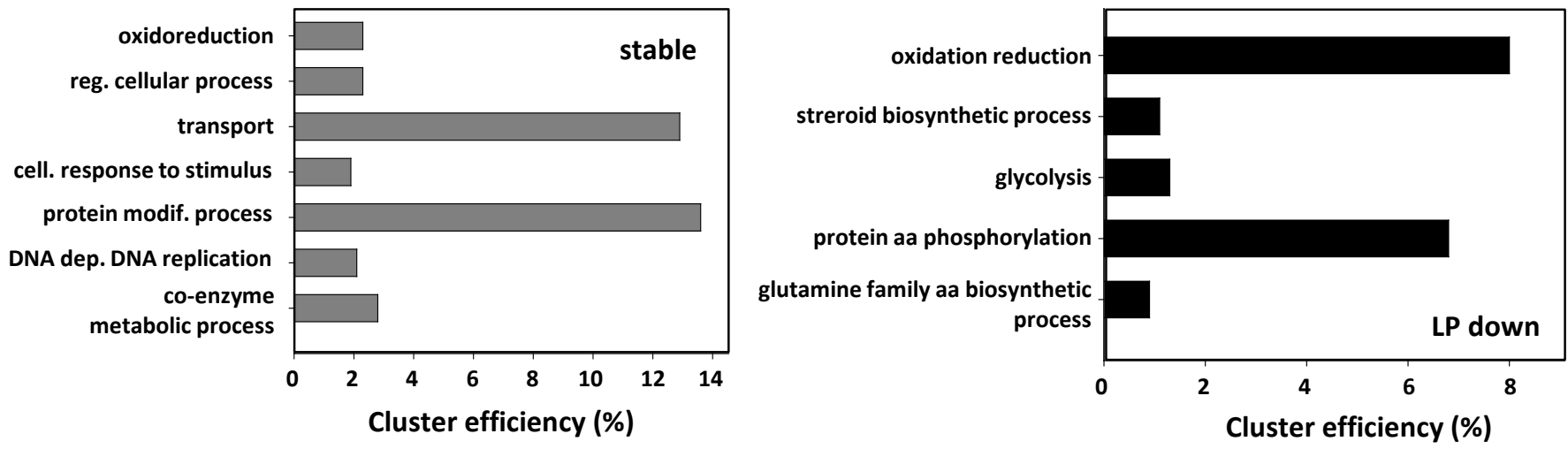

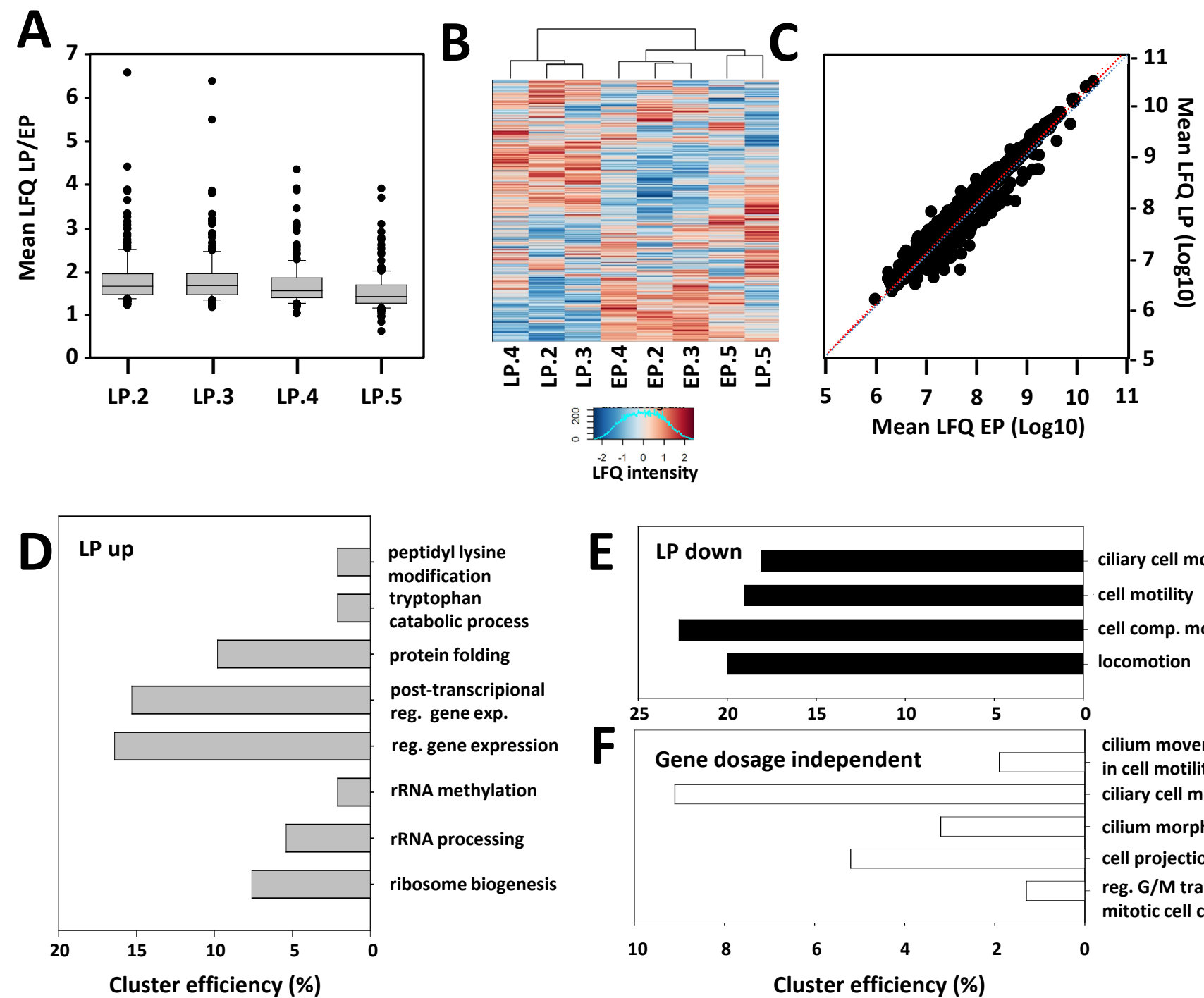

ciliary cell motility cell motility

ell comp. movement locomotion

Cluster efficiency (\%)

Cluster efficiency (\%)

\begin{tabular}{|c|c|c|c|c|}
\hline \multirow{20}{*}{ 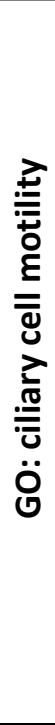 } & $\frac{\text { LD1S_360789100 }}{\text { LD1S } 360785400}$ & $\frac{\text { rib72 protein-like protein }}{\text { centrin-3 }}$ & $E$ & \multirow[t]{2}{*}{ Protein } \\
\hline & LD1S 360774100 & Coiled-coil domain containing 147. Function unknown & & \\
\hline & LD1S_360729600 & dynein heavy chain (pseudogene), & & DINA \\
\hline & LD1S_350712400 & PACRGB & $=$ & \\
\hline & LD1S_350682700 & nexin-dynein regulatory complex 4 & & \\
\hline & LD1S_340658700 & dynein heavy chain & E & \\
\hline & LD1S_290419900 & flagellar radial spoke protein-like & & \\
\hline & LD1S_280411200 & dynein heavy chain & 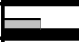 & \\
\hline & LD1S_280385600 & dynein heavy chain & $\overline{\bar{E}}$ & \\
\hline & LD1S_270379000 & Coiled-coil domain containing 147. & & \\
\hline & LD1S_270378700 & dynein heavy chain & & \\
\hline & LD1S_270374300 & WD domain, G-beta repeat & $=$ & \\
\hline & LD1S_270370400 & paraflagellar rod protein 5 & & \\
\hline & LD1S_250306600 & dynein heavy chain & & \\
\hline & LD1S_240273900 & Dynein intermediate chain 1 , axonemal & & \\
\hline & LD1S_220241800 & IQ motif and ubiquitin domain containing & & \\
\hline & LD1S_160127400 & WD domain, G-beta repeat & & \\
\hline & LD1S_140088200 & dynein heavy chain & & \\
\hline & LD1S_130074700 & ATPase family associated with various cellular activities (AAA) & & \\
\hline & LD1S 130063300 & Flagellar radial spoke protein $4 / 6$ & & \\
\hline
\end{tabular}




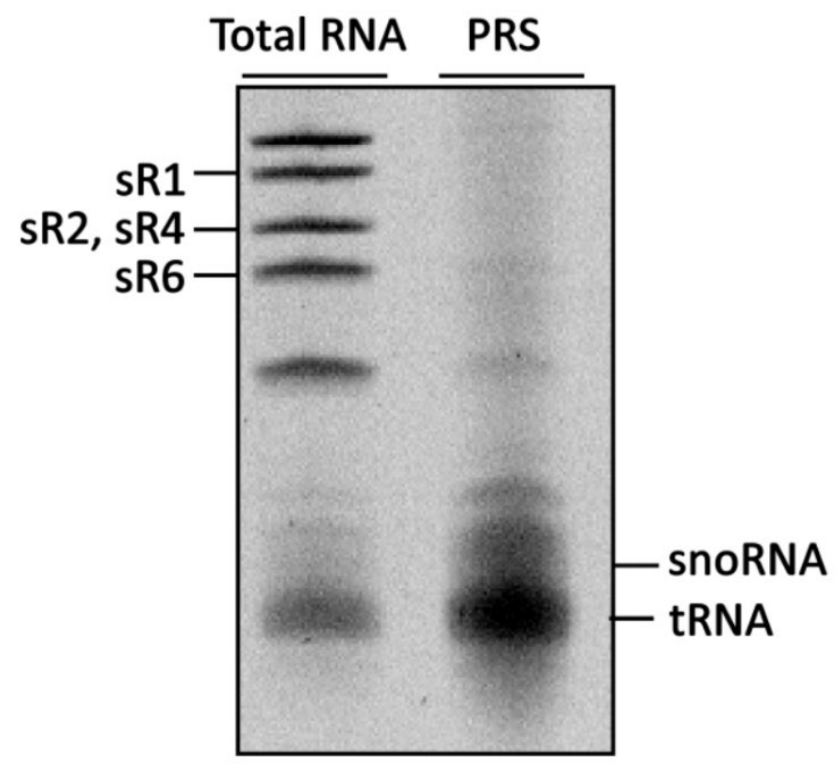

Figure S7 